minosen u. s. w. genau festzustellen, um mit dieser Methode die Stärke in den verschiedenen Mehlsorten und stärkemehlhaltigen Nahrungsmitteln quantitativ bestimmen $\mathrm{zu}$ können, worüber einige Vorversuche zu einem günstigen Resultate führten, auch wird die Einwirkung von Zinkchlorid auf Kaseïn, Eiweissstoffe u. s. w. näher verfolgt und über das Ergebniss demnächst berichtet werden.

\title{
Neue Verfahren zur Reinigung von Schmutzwässern.
}

Toll

\section{J. König (Rof.). E. Hasplhoff nnd R. Grossunann in Mïnster i. W.}

Zur Reinigung von Schmutzwässern, besonders von städtischen $A$ bwässern (Spuiljauche), sind in den letzten Jahren verschiedene neue Verfahren in Vorschlag gebracht und vielfach in den Zeitschriften wie Zeitungen mit und ohne Verständniss besprochen worden; es sind dieses:

1. Das elektrisehe Reinigungsverfahren.

Man unterscheidet hierbei das Verfahren vou W. Webster, bei dem der elektrische Strom unter Anwendung von Fisenplatten alf das chloridbaltige oder mit Chloriden versetzte Wasser einwirkt, und das Verfabren von E. II crmite $u$. $\Lambda$., bei dem Platin- und Zinkplatten odır Kchlı- und Zinkplatten als Elektrod'n dienen.

In ersterom Falle wirkt das aus dי.m Chlorid abgeschiedene Chlor lïsend auf die positive Eisenplatte; es bildet sich Ferrocblorid, wclches durch das unter Entwickelung von Wasscrstofi an dem negativen Pol gebildete Mutallhydroxyd - also Natriumhydroxyd bei Anwesenhrit von Chlornatrium - sofort in das betreffende Metallelılorirl (also Natriumeblorid) und Fcrrolydroxyd zerlegt wird.

Bei Anwendung von Hatin oder Kohle als Anode nach dem Hermite-Verfahren kann das aus dem Chlorid durch den elektrischen Strom freigemachte Chlor nicht lösend auf die Elcktrode wirkın; es tritt entweder als fruies Chlor oder als unterchlorige Säur' oder unterchlorigsaures Salz auf.

Das Webster'sche Reinigungsverfabren ist daher, wie der Erste von uns in Gemeinschaft mit (. Remelí) gezeigt hat, nichts weiter als ein ehemisches Reinigungsverfahren, nur mit dem Unterschiede, dass das Metallhydroxyd (hier Ferrohydroxyd), welches fallend auf die Schwebestoffe des Schmutzwassers wirkt, fortgesetzt durch den elektrischen Strom erzeugt wird, währond dasselbe bei der üblichen chemischen Reinigung durch Zusatz von fertig gebildetem Ferrosulfat und Kalk entsteht. Sonstige ehemische Nebenwirkungen, besonders oxydirende Nebenwirkungen, wie dieses behauptet worden ist, übt der elektrische Strom nicht aus; im Gegentheil wirkt dersclbe, wenn nur Chloride als

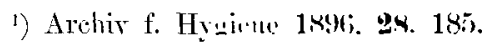


leitende Salze angewendet werden, in Folge der fortwährenden Wasserstoffentwickelung eher reducirend. Dagegen hat das Webster'sche elektrische Reinigungsverfahren den Vorzug, dass die Flüssigkeit, wenn sie neutral war, stets neutral bleibt, weil die Umsetzung stöchiometrisch verläuft, während bei dem chemischen Reinigungsverfahren sich durchweg kaum ein Ueberschuss von freiem Alkali (Kalk) vermeiden lässt.

Das Hermite-Verfahren ist dagegen nichts anderes als ein Desodorationsoder Desinfektionsverfahren, indem das unterchlorigsaure Salz einerseits den üblen Geruch der Abortjauche beseitigen, andererseits die weitere Zersetzung hintanhalten soll.

Die mit beiden Verfahren angestellten Versuche haben bis jetzt zu günstigen oder im Grossen praktisch verwendbaren Ergebnissen nicht geführt und werden auch wohl nur da in Betracht kommen können, wo bessere Verfahren nicht durchführbar sind oder eine billige Elektricitätsquelle zur Verfügung steht ${ }^{1}$ ).

\section{Das Ferrozone-Polarite-Verfahren,}

Dasselbe besteht darin, dass das Abwasser zunächst mit Ferrozone (schwefelsaure Thonerde und schwefelsaures Eisenoxyd in wechselnden Mengen) versetzt und das geklärte Wasser durch ein Filter von Sand und Polarite (im wesentlichen Eisenoxyd) filtrirt wird.

Der Zusatz von Ferrozone soll eine Fällung der Schwebestoffe, die Filtration durch die Sand-Polarite-Schicht eine starke Oxydation bewirken, indem der gebundene Sauerstoff des Eisenoxyds des Polarites zur Oxydation verwendet werden soll.

Diese Ansicht hat anscheinend durch mehrere Versuche ihre Bestätigung gefunden.

Was zunächst die Zusammensetzung der beiden Reinigungsmittel anbelangt, so scheint dieselbe nach folgenden Analysen verschieden zu sein.

1. Ferrozone.

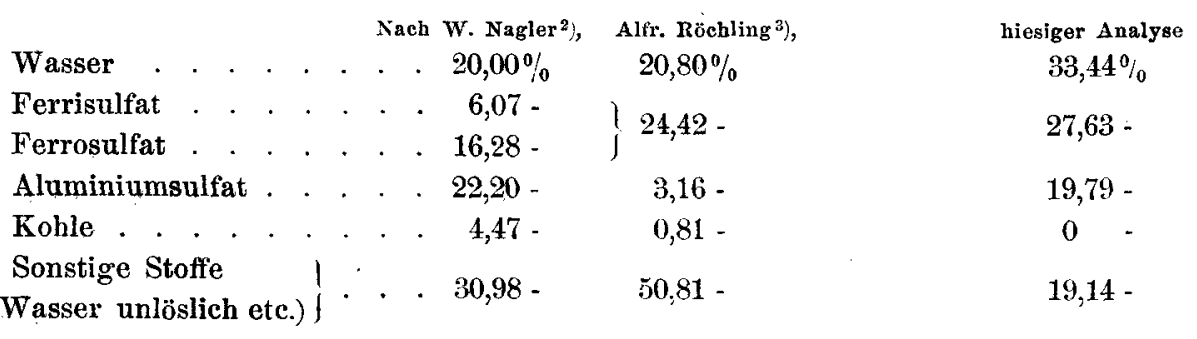

1) Wenn Herr Dr. W. Bersch in einem Berieht über vorstehende Arbeit im Centr.-Bl. $\mathrm{f}$. Agrik.-Chem. 1897, 26, 774 sagt: „dass die Wirksamkeit und Anwendbarkeit dieses Verfahrens ausser allem Zweifel steht", so scheinen ihm die Versuchsergebnisse uber diese Verfahren nicht sämmtlich bekannt geworden zu sein.

2) Chem. Centr.-Bl. 1894, I, 1086.

3) Gesundheits-Ingenieur 1892, 15, 585. 
2. Polarite.

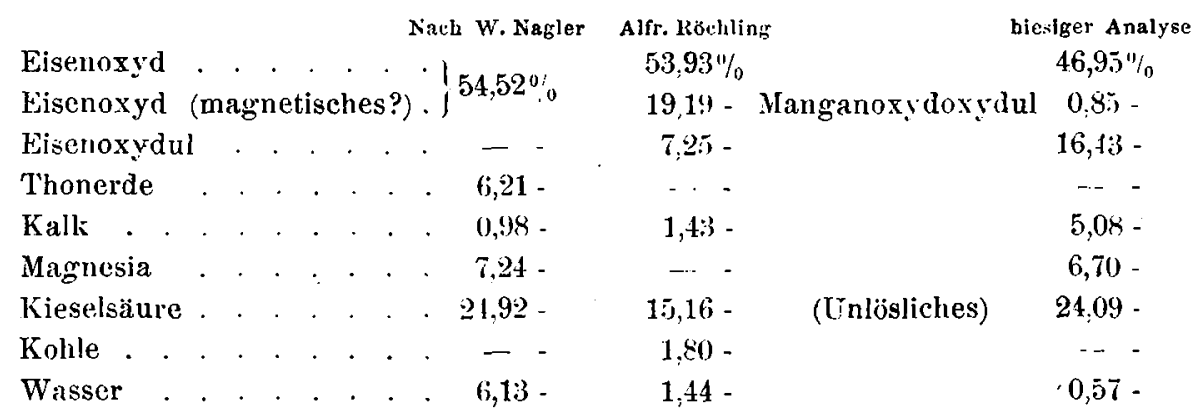

Nach den ersten Berichten von W. Nagler und H. Alfr. Röchling (l. c.) hat das Verfahren in England gute Erfolge gehabt; es sind durch das Fällungsmittel Ferrozone nicht nur bis $80 \%$ des organischen Stickstofts aus den städtischen Abwässern entfernt, es soll auch in den Polarite-Filtern eine wesentliche Oxydation der gelösten organischen Stoffe und cinc: Bildung von Salpetersäure aus dom Ammoniak bewirkt worden sein.

J. H. Vogel') untrersuchte die Wirkung des Verfabrens in den Anlagen in Heudon (mit 16000 Einwohnern), Acton (mit 30000 Einwolnern) und Boyton (mit 13500 Einwohneru). Die Abwässer - olme Einschluss von hegenwasser, welches in England für sich abgeleitet zu werden pflegt - wurden auf 5-10000 l mit $1 \mathrm{~kg}$ Ferrozone versetzt, nach inniger Durchmischung in grossen Klärbecken behufs Klärung 4 Stunden der Ruhe überlassen und das geklärte Wasser dureh die Polarite-Filter filtrirt, welche von unten nach oben folgend gobildet worden waren aus einer Schicht von: $10-15 \mathrm{~cm}$ kleinen Feldsteinen und grobem Kies, $10 \mathrm{~cm}$ Sand, $30 \mathrm{~cm}$ Polarite (erbsengrosse Stücke) und $25--30 \mathrm{~cm}$ scharfem Sand (vergl. Fig. 1\%); die Filtrirkammern hatten 20-30 m Länge und 10-15 m Breite; in einer Tiefe von $1 \mathrm{~m}$ unter den Feldsteinen waren Drainrohre angebracht; auf 1 qm Filter kamen $200 \mathrm{~kg}$ Polarite; im Durchschnitt filtrirten $30-45 \mathrm{hl}$ pro $1 \mathrm{qm}$ und Tag. Nach 4 wöchentlichem Betriebe wurden die Filter unbrauchbar; sie blieben dann 8 Tage unbenutzt stehen, um durch den I uftsauerstoff regenerirt und wieder betriebsfuhig zu werden.

Für den Stickstoffgehalt der ungereinigten und gercinigten Wässer fand J. H. Vogel folgende. Zahlen für 11 :

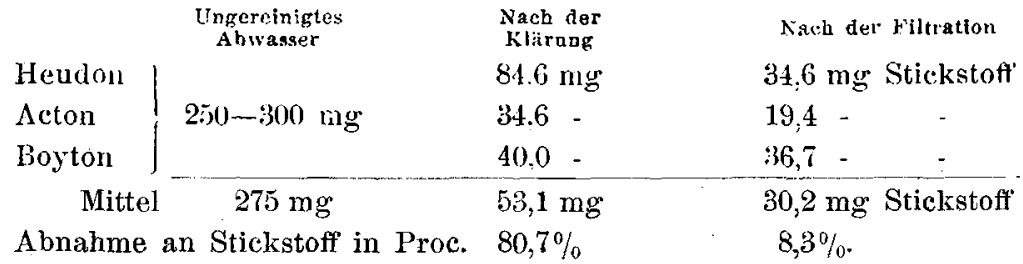

In einem anderen Versuch hatte in Heudon durch die Klärung und Filtration der Abdampfrückstand um 86\%, der Glührückstand um 72\% abgenom-

1) Nach einem mir vom Verf. gütigst überlassenen Reisebericht. 
men. Das Wasser floss klar, farb- und geruchlos ab. Das sind äusserst günstige Ergebnisse, die aber wohl zum Theil auf die starke Koncentration des Abwassers - bei Einschluss von Regenwasser oder in grosser Verdünnung hält die Reinigung an sich schwieriger - zurückgeführt werden müssen.

Jedoch fand J. H. Vogel die Behauptung, dass durch dieses Verfahren Ammoniak zu Salpetersäure oxydirt werde, nicht bestätigt.

In der Sonderausschuss-Sitzung der deutschen Landwirthschafts-Gesellschaft am 17. Februar 1897 hat jedoch J. H. Vogel ${ }^{1}$ ) erklärt, dass nach den inzwischen in Bromberg angestellten Versuchen das Ferrozone-Polarite-Verfahren eine energische, der Wirkung der Rieselfelder gleichkommende Oxydation bewirke.

Das in Bromberg angewendete Filter hatte nach einem Bericht von Metzg er ${ }^{2}$ ) in der Höhe eine gleiche Anordnung, wie sie Fig. 12, das von uns benutzte Filter darstellt. Das städtische Abwasser wurde auf $1000 \mathrm{l}$ mit $100 \mathrm{~g}$ Ferrozone (mit $67,46 \%$ Aluminiumsulfat, $8,41 \%$ Eisensulfat und 5,84\% freier Schwefelsäure) in einem Klärbecken innig vermischt, 2 Stunden der Ruhe überlassen und das geklärte Wasser auf das Filter geleitet, welches für die Abgänge von 300 Menschen = etwa 250001 4,5 qm gross war.

Im Mittel von 18 Einzelversuchen in den Tagen vom 13. Oktober bis 4. November 1896 wurde gefunden:

\begin{tabular}{cc|ccc|cc}
\multicolumn{2}{c|}{ Rohe Jauche } & \multicolumn{2}{c}{ Geklärte Jauche } & \multicolumn{2}{c}{ Filtrat } \\
$\begin{array}{c}\text { Organische } \\
\text { Substanz }\end{array}$ & Keime: & $\begin{array}{c}\text { Organische } \\
\text { Substanz }\end{array}$ & Keime & $\begin{array}{c}\text { Organische } \\
\text { Substanz }\end{array}$ & Keime \\
$665,6 \mathrm{mg}$ & 535194 & $275,1 \mathrm{mg}$ & $\mathbf{1 5 2 4 8 3}$ & $184,1 \mathrm{mg}$ & 38995
\end{tabular}

In diesen Untersuchungen ist leider der wichtigste Bestandtheil, der Stickstoff, nicht berücksichtigt und die Frage geprüft worden, welche Umwandlungen derselbe hierbei erfährt und ob die Polarite-Masse wirklich die Bildung von Salpetersäure begünstigt. Die Abnahme an organischen Stoffen in vorstehenden Versuchen braucht ebenfalls noch nicht auf eine wirkliche Oxydation durch das Polarite zurückgeführt zu werden; sie kann auch auf einer mechanischen Wirkung des Filters beruhen.

Da nach unseren jetzigen Anschauungen die Oxydation des Kohlenstoffs wie des Stickstoffs der organischen Stoffe zu Kohlensäure bezw. zu Salpetersäure durch Vermittelung von Bakterien erfolgt, welche den freien Sauerstoff der Luft auf die organischen Stoffe übertragen, so verdiente die Frage, ob der gebundene Sauerstoff der Polaritemasse (des Eisenoxyds ete.) wirklich eine Oxydation der organischen Stoffe bewirkt oder doch begünstigt, einer erneuten Prüfung.

Wir haben für den Zweck eine Reihe von Versuchen angestellt, indem wir die Wirkung des Polarite-Filters im Vergleich zu einem solchen aus Ackerboden, Koks und Calciumorthoplumbat (Kassner's) ermittelten. Letzteres wurde deshalb mit hinzúgezogen, weil dasselbe nach den Untersuchungen G. Kassner's

1) Nach dem mir vorliegenden Protokoll.

2) Gesundheits-Ingenieur, 1897, 20, 1. 
leicht Sauerstoff abgiebt, daher in ähnlicher Weise wirken müsste, wie diesos von dem Polarite behauptet wird. Es wurde in nussgrossen Stücken verwendet, ebenso wie das Polarite und der Koks. Das Ferrozone wie Polarite wurde uns bereitwilligst von der International-Water \& Sewage Purification in London zur Verfügung gestellt. Als Boden verwendeten wir sandigen Gartenboden in alter Kultur, in welchem nitrificirende Baktcrien vorausgesetzt werden konnten.

Die Filter in Blecheylindern von $40 \mathrm{~cm}$ Durchmesser hatten nach den in England und Bromberg angewendeten Mustern die nachstehende .norinung (Fig. 1:):

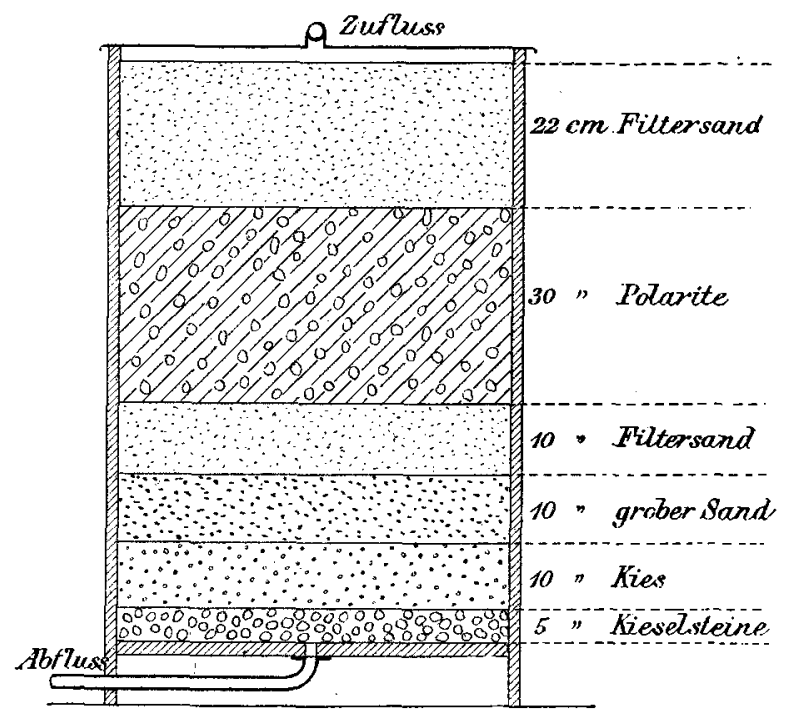

P.i. 12.

Polaritu-Filter.

Die Vergleichsfilter hatten für Koks und Calciumplumbat genau dieselbe Anordnung, nul wit dem Enterschiede, dass sie statt der $30 \mathrm{~cm}$ hohen Schicht Polarite dieselbe IJöhenschicht Koks bezw. Calciumorthoplumbat (letzteres gemischt mit Kies) enthielten. Nur bei dem Bodentilter lag die $30 \mathrm{~cm}$ hohe Bodenschicht oben, dann folgte Kies, Sand etc. wie bei den andern Filtern. Als Schmutzwasscr wurde verdünnte Abortjauche verwendet, die auf etwa 7501 mit $200 \mathrm{~g}$ Ferrozone versetzt, gut durchgerülırt und in einem grösseren Bottich behufs Klärung einige Zcit der liuhe überlassen wurde. Das geklärte Wasser wurde dann gleichmässig und zn gleicher Zcit troptenweise oder in dünnem Strahl durch die Filter filtrirt, das Filtrat in klcinen Behältern gesammelt und nach dem Durchmischen gleichmässig mit der geklärten Jauche untersucht. Zwischen dın einzelnen Filtrationsversuchen lag meistens eine Ruhepause von einem oder mohreren Tagen, zwischen Versuch 5 und 6 eine solche von 14 Tagen. Die liestimmung des Stickstoffs in den einzelnen Verbindungstormen erfolgte, wie weiter unten S. 182 angegeben ist. Die Untersuchung ergab folgende Resultate füir 1 l: 


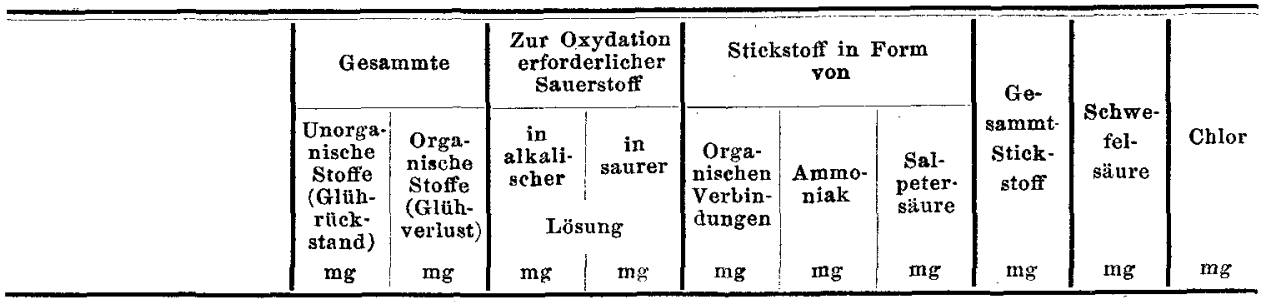

1. Versuch.

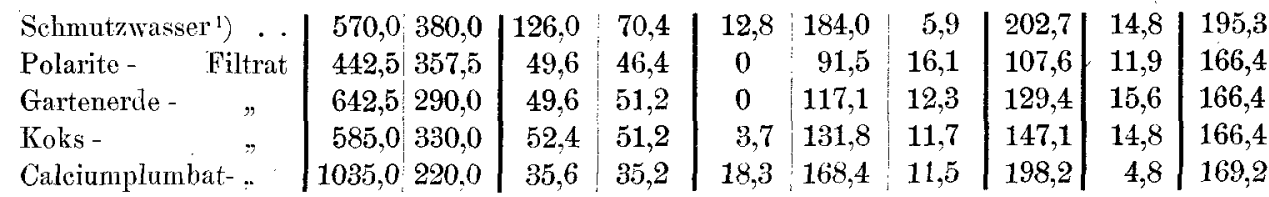

2. Versuch.

Schmutzwasser:) . Polarite - Filtrat Gartenerde - .. Koks Calciumplumbat- .. $\quad 1097,5,172,5$

\begin{tabular}{r|r|l|r|r|r|r|r}
49,6 & 60,8 & 7,3 & 109,8 & $\mathbf{6 , 2}$ & 123,4 & 32,6 & $\mathbf{1 2 3 , 9}$ \\
$\mathbf{2 5 , 6}$ & $\mathbf{2 7 , 2}$ & 0 & $\mathbf{9 6 , 9}$ & $\mathbf{1 6 , 5}$ & $\mathbf{1 1 3 , 5}$ & $\mathbf{6 8 , 1}$ & $\mathbf{1 1 6 , 8}$ \\
38,0 & 38,4 & 0 & $\mathbf{7 6 , 9}$ & $\mathbf{2 4 , 2}$ & 101,1 & 66,2 & $\mathbf{1 1 6 , 8}$ \\
33,6 & $\mathbf{3 7 , 6}$ & 0 & $\mathbf{1 0 6 , 1}$ & 26,7 & $\mathbf{1 3 2 , 9}$ & $\mathbf{5 6 , 9}$ & $\mathbf{1 2 3 , 9}$ \\
36,8 & 32,0 & 3,7 & $\mathbf{1 4 6 , 4}$ & $\mathbf{7 , 3}$ & $\mathbf{1 5 7 , 4}$ & 5,2 & $\mathbf{1 0 2 , 6}$
\end{tabular}

3. Versuch.

\begin{tabular}{lc|r|r|} 
Schmutzwasser') & $\cdots$ & 422,5 & 270,0 \\
Polarite - Filtrat & 347,5 & 270,0 \\
Gartenerde - &.. & 465,0 & 300,0 \\
Kolks - &. & $\mathbf{5 1 0 , 0}$ & 290,0 \\
Calciumplumbat-.. & 1397,5 & 27,5
\end{tabular} \begin{tabular}{r|l|l|r|r|r|r|r}
$\mathbf{6 1 , 2}$ & 65,6 & 5,9 & $\mathbf{1 2 2 , 2}$ & 9,2 & 137,3 & $\mathbf{6 5 , 5}$ & 113,3 \\
42,4 & 47,2 & $\mathbf{4 , 5}$ & 86,0 & 28,9 & $\mathbf{1 1 9 , 5}$ & 52,8 & 113,3 \\
47,2 & 43,2 & 1,8 & 89,7 & 24,7 & 116,2 & $\mathbf{5 4 , 9}$ & $\mathbf{1 1 3 , 3}$ \\
39,6 & $\mathbf{4 3 , 2}$ & 0 & 86,0 & $\mathbf{4 0 , 4}$ & $\mathbf{1 2 6 , 4}$ & $\mathbf{7 0 , 3}$ & 109,7 \\
32,0 & 30,4 & 3,7 & $\mathbf{1 1 3 , 5}$ & 8,2 & $\mathbf{1 2 5 , 3}$ & $\mathbf{1 6 , 1}$ & 113,3
\end{tabular}

4. Versuch.

\begin{tabular}{lc|r|r|r|} 
Schmutzwasser ') &. & 320,0 & 102,5 \\
Polarite- & Filtrat & 302,5 & 115,0 \\
Gartenerde- & $*$ & 410,0 & 202,5 \\
Koks - &. & 405,0 & 367,5 \\
Calciumplumbat- .. & $\mathbf{1 4 9 5 , 0}$ & $\mathbf{7 2 , 5}$
\end{tabular}
\begin{tabular}{l|l}
25,6 & 22,4 \\
\hline
\end{tabular} \begin{tabular}{l|l}
26,4 & 20,8
\end{tabular} \begin{tabular}{ll}
26,8 & 20,0 \\
\hline
\end{tabular} \begin{tabular}{|l|l|}
25,2 & 23,2
\end{tabular}

\begin{tabular}{l|r|r|r}
7,2 & 54,3 & 5,8 & 67 \\
$9, \mathbf{1}$ & 70,6 & 14,5 & 94 \\
0 & 56,1 & 23,2 & 79 \\
0 & 56,1 & 45,3 & 101 \\
5,4 & 90,5 & 6,9 & 102
\end{tabular}

67,3
94,1
79,3
101,4
102,8

45,6 70,8

5. Versuch.

\begin{tabular}{|c|c|c|}
\hline & \begin{tabular}{ll|l}
402,5 & 115,0
\end{tabular} \\
\hline \multicolumn{2}{|c|}{ Polarite - Filtrat } & $\begin{array}{lll}442,5 & 307,5\end{array}$ \\
\hline Gartenerde - & .. & \begin{tabular}{ll|l}
680,0 & 487,5
\end{tabular} \\
\hline Koks - & & \begin{tabular}{|l|l|l|}
517,5 & 340,0
\end{tabular} \\
\hline Calciumplumb & bat- & $1197,5 \quad 77.5$ \\
\hline
\end{tabular}

\begin{tabular}{|c|c|c|r|r|}
30,0 & 28,0 & 10,1 & 47,8 & 4,7 \\
31,2 & 28,8 & 0 & 28,9 & 44,5 \\
36,4 & 34,4 & 0 & 21,7 & 78,2 \\
24,8 & 23,2 & 0 & 21,7 & 48,2 \\
25,6 & 21,6 & 5,4 & 59,7 & $\mathbf{7 , 2}$
\end{tabular}

\begin{tabular}{r|r|}
62,6 & 101,2 \\
73,5 & 102,0 \\
99,9 & 91,8 \\
69,9 & 87,5 \\
72,4 & 24,0
\end{tabular}

53,1 70,8 56,6 53,1 63,7

6. Versuch. (Nach 14 tägiger Pause ausgeführt.)

\begin{tabular}{|c|c|c|c|c|c|c|c|c|c|c|}
\hline Schmntzwasse & & \begin{tabular}{l|l|l}
295,0 & 175,0
\end{tabular} & 25,2 & 23,2 & 14,9 & 54,4 & 4,4 & 73,6 & $13 \tilde{5}, 5$ & 120,4 \\
\hline Polarite - & Filtrat & \begin{tabular}{l|l}
470,0 & 470,0
\end{tabular} & 24,4 & 22,4 & 3,7 & 7,2 & 43,7 & 54,7 & 1355,5 & 120,3 \\
\hline Gartenerde- & . & $595,0 \quad 530.0$ & 25,6 & 24,0 & 0 & 16,5 & 61,2 & 77,6 & 137,2 & 116,8 \\
\hline Koks - & .- & $455,0 \quad \mathbf{4 4 5 , 0}$ & 28,0 & 27,2 & 0 & 11,6 & 42,9 & 54,5 & 150,9 & 120,4 \\
\hline Calciumplum & at- . & $1120,0250,0$ & 26.0 & 25,6 & 9,4 & 52,5 & 7,6 & 69,5 & 36,9 & 134,5 \\
\hline
\end{tabular}




\begin{tabular}{|c|c|c|c|c|c|c|c|c|c|c|}
\hline \multirow[t]{3}{*}{. } & \multicolumn{2}{|c|}{ Gesammte } & \multicolumn{2}{|c|}{$\begin{array}{l}\text { Zur Oxydation } \\
\text { erforderlicher } \\
\text { Sanerstoff }\end{array}$} & \multicolumn{3}{|c|}{$\begin{array}{c}\text { Stickstoff in Form } \\
\text { von }\end{array}$} & \multirow{3}{*}{$\begin{array}{c}\text { Ge- } \\
\text { sammt- } \\
\text { Stick- } \\
\text { stoff }\end{array}$} & \multirow[b]{2}{*}{$\begin{array}{l}\text { Schwe- } \\
\text { fel- } \\
\text { säure }\end{array}$} & \multirow[b]{2}{*}{ Chlor } \\
\hline & $\begin{array}{l}\text { Anorga- } \\
\text { nische } \\
\text { Stoffe } \\
\text { (Gliuh- } \\
\text { rück- } \\
\text { stand) }\end{array}$ & $\begin{array}{c}\text { Orga- } \\
\text { nische } \\
\text { Stoffe } \\
\text { (Glüh- } \\
\text { verInst) }\end{array}$ & $\begin{array}{c}\text { in } \\
\text { alkali- } \\
\text { scher } \\
\text { Lö }\end{array}$ & $\begin{array}{l}\underset{\text { in }}{\text { saurer }} \\
\text { ing }\end{array}$ & $\begin{array}{c}\text { Orga- } \\
\text { nisehen } \\
\text { Verbin- } \\
\text { dungen }\end{array}$ & $\underset{\text { niak }}{\text { Ammo- }}$ & $\begin{array}{l}\text { Sal- } \\
\text { peter- } \\
\text { säure }\end{array}$ & & & \\
\hline & $\mathbf{m g}$ & $\mathrm{mg}$ & $\mathbf{m g}$ & $\mathrm{mg}$ & mg & $\mathrm{mg}$ & $\mathbf{m g}$ & & $\mathbf{m g}$ & $\mathbf{m g}$ \\
\hline
\end{tabular}

7. Vorsuch.

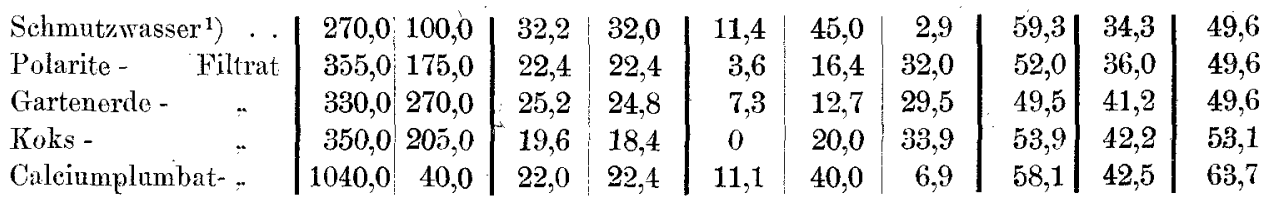

Mittel aus vorstehenden 7 Versuchen.

\begin{tabular}{|c|c|c|c|c|c|c|c|c|c|}
\hline Schmutzwasser') . & $377,1 \mid 201,8$ & 50,1 & 43,2 & 9,9 & 88,2 & 5,6 & 103,8 & 61,4 & 103,7 \\
\hline Polarite - Filtrat & $\begin{array}{ll}374,3 & 261,8\end{array}$ & 28,5 & 27,8 & 2,4 & 54,5 & 23,5 & 80,4 & 60,1 & 93,5 \\
\hline Gartenerde - & $521,4340,4$ & 35,5 & 33,8 & 1,3 & 55,8 & 36,2 & 93,3 & 65,8 & 98,6 \\
\hline Koks - & $470,0 \quad 316,4$ & 32,1 & 31,5 & 0,7 & 61,9 & 35,6 & 98,0 & 67,8 & 99,6 \\
\hline Calciumplumbat-.. & \begin{tabular}{ll|l}
1197.6 & 137.1
\end{tabular} & 29,0 & 27,2 & 8,1 & 95,2 & 7,9 & 111.9 & 20,7 & 102,5 \\
\hline
\end{tabular}

Aus vorstehenden Untersuchungen ersehen wir, dass die filtrirten Wässer an gesammten löslichen Stoffen, unorganischen wie organischen, d. h. Glührückstand und Glühverlust im Anfang zwar weniger, nachher aber - mit Ausnahme bei dem Calciumplumbatfilter - erheblich mehr enthalten, als das ursprüngliche, geklärte Jauchewasser. Das erklärt sich daraus, dass die trocknen oder die in der Ruhezeit austrocknenden Filter einerseits Jauehewasser und Jauchestoffe zurückhalten, die sich bei einer späteren Filtration wieder lösen, dass andererseits die Filter an sich lösliche Stoffe enthalten oder dass die gebildeten Oxydationsprodukte (Kohlensäure, Salpetersäure etc.) lösend auf Bestandtheile der Filter wirken (vergl. auch weiter unten). Der Verbratich an Kaliumpermanganat oder an zur Oxydation erforderlichem Sauerstoff nimmt in allen Versuchen mehr oder weniger $a b$, ein Beweis, dass bei der Filtration eine Oxydation der organischen Stoffe stattgefunden hat. Auch die Salpetersäure hat in allen Versuchen im Anfange eine geringere, später eine grössere Zunahme erfahren; dasselbe gilt im geringeren Maasse von der Schwefelsäure; im Allgemeinen kann auch hier eine geringe Zunahme festgestellt werden. Eine Ausnahme bildet nur das Filter mit der Calciumplumbatschicht; hierin nimmt die Salpetersäure garnicht oder nur unerheblich $z u$, die Schwefelsäure sogar ab; letztere Abnahme kann nur dadurch erklärt werden, dass das Calciumplumbat zersetzt und unlösliches schwefelsaures Blei gebildet wird. Die Bildung der Salpetersäure muss. wenigstens auf eine Bakterienwirkung zurückgeführt werden und spielt hierbei obne Zweifel der gebundene Sauerstoff des Eisenoxyds im Polarite keine Rolle; denn in dem Polaritefilter ist die gebildete Salpetersiure durchweg geringer als bei den Filtern aus Gartenerde und Koks. Dass die Salpetersäurebildung wesentlich von nitri-

1) Vergl. Anm. 1 S. 176. 
ficirenden Bakterien bedingt wird, kann daraus geschlossen werden, dass diese mit der Länge der Gebrauchszeit, also nachdem sich die Bakterien mehr und mehr in den Filtern entwickelt haben, zunimmt, ferner daraus, dass in dem Filter von Calciumplumbat die Salpetersäurebildung gleich Null oder nur eine ganz geringe ist, obschon dieses noch leichter den gebundenen Sauerstoff abgiebt als das Eisenoxyd des Polarites. Es ist anzunehmen, dass durch die geringen, in Lösung gegangenen Mengen Blei die Nitrifikationsbakterien vernichtet worden sind. Auch haben wir in dem Polaritefilter keine Reduktion des Eisenoxyds zu Eisenoxydul nachweisen können. Man kann hiergegen vielleicht einwenden, dass das gebildete Eisenoxydul in den Filtern durch Luftzutritt alsbald wieder in Eisenoxyd übergeführt wird. Wir haben aber in luftdicht verschlossenen Flaschen Jauche mit Polaritemassen unter öfterem Umschüttel längere Zeit stehen lassen, aber auch keine Reduktion feststellen können. Eine solche ist allerdings im Boden bei Abschluss von Luft unter dem Einfluss von Bakterien anzunehmen; indess fragt sich, ob das Eisenoxyd hierbei nicht vorher durch eine Săure gelöst bezw. gelockert werden muss, oder ob sich Eisenoxydhydrat, wie es im Boden vorhanden zu sein pflegt, nicht anders verhält als das Eisenoxyd des Polarites.

Dass fein vertheilter oder auf der rauhen Oberfläche eines Körpers mechanisch verdichteter Sauerstoff die Nitrifikation unterstützt, dürfte daraus zu folgern sein, dass das Filter von Koks eine im Durchschnitt gleiche Menge Salpetersäure gebildet hat als das mit Gartenerde. Diese Art Sauerstoff kann aber mit dem chemisch gebundenen des Eisenoxyds zur Befriedigung des Sauerstoffbedürfnisses der nitrificirenden Bakterien nicht verglichen werden.

Die Menge des Gesammtstickstoffs in den Filtraten ist ja nach den in den Filtern zurückgehaltenen Mengen Stickstoff in den einzelnen Versuchen bald geringer, bald grösser, im Durchschnitt der 7 Versuche bei den Filtern aus Polarite, Gartenerde und Koks geringer, bei dem Filter aus Caleiumplumbat aber nahezu gleich oder sogar etwas höher ${ }^{1}$ ), als der des unfiltrirten Jauchewassers für gleiches Volumen (nämlich für $1 \mathrm{l}$ ). Daraus folgt, dass bei der Nitrifikation des Stickstoffs auch freies Stickstoffgas entstehen muss, was mit den folgenden und anderweitigen Versuchen übereinstimmt.

Da die Abnahme im Verbrauch an Kaliumpermanganat oder dem zur Oxydation erforderlichen Sauerstoff in den Filtraten von den Polarite- und Calciumplumbatfiltern eine gleiche und im Durehschnitt der 7 Versuche sogar eine etwas grössere ist als bei dem Boden- und Koksfilter, so scheint die Oxydation von leicht oxydirbaren organischen Kohlenstoffverbindungen weniger von Mikroben als von genügendem, fein vertheiltem bezw. leicht abspaltbarem Sauerstoff abhängig zu sein.

Hierüber sollen aber nach längerer Ruhezeit der Filter noch weitere Versuche angestellt werden.

1) Die Differenz muss wohl anf Ungleichheit einer oder einiger Proben des Filtrats in Folge zurückgehaltener Stoffe von den vorhergehenden Versuchen tiegen. 
3. Reinigung durch Fäuluiss, Lüftung und Oxydation (Filtration).

Neuerdings ist von W. J. Dibdin ${ }^{\prime}$ ) in London der Vorschlag gemacht, die stiadtischen Abwässer durch Fäulniss, darauf folgende Lüftung und Oxydation verbunden mit Filtration zu reinigen. Statt die Fäulniss zu beseitigen oder zu verhindern, wird dieselbe unterstuitzt, indem man das Abwasser mit stark in Fäulniss begriffenem Wasser gleichsam impft, cine gewisse Zeit (bis zu 24 Stund'n) faulen lässt, dann lüftet, oxydirt und durch ein Koks-Sandfilter filtrirt, worin eine Oxydation der organischen Stoffe vor sich geht.

Dieser Vorschlag ist nicht neu. Schon vor 12 Jahren hat sich $\Lambda$ lex. Mülle $\mathbf{r}^{1}$ ) rin Verfalıren patentiren lassen, wonach die mit organischen Stoffen (vorwiegend Zuckerfabrikabwässer) in Erdgruben nach Erwärmen auf 25-40 unter Zusatz von liefeartigen Organismen der Gährung bezw. Fäulniss überlassen und dann durch Filter von Sand, Kohle ete. filtrirt werden sollen.

Walter East") empfahl cbenfalls um dieselbe Zeit, dic Kloakenwässer durch Zusatz von stark faulenden Flüssigkeiten in eine beschleunigte Füulniss zu versetzen, die hierbei auftretenden Fäulnissgase durch cine Fisenoxydschicht nach cinem Schornstein abzuführen, in die gefaulte Flüssigkeit Luft zu pressen, alsclann dieselbe nach der Filtration zur Berieselung zu verwenden.

An sich können diese Vorschläge als zweckdienlich bezcichnet werden. Bei den mit organischen Stoffen verunreinigten Schmutzwässern müssen die organischen Verbindungen nämlich, wenn sie wie gewöhnlich nicht ausgefällt werden könnem, auf irgend eine Weise zerlegt und entweder vergast oder mineralisirt, d. h. in unschädliche bezw. unschädlichere Verbindungen übergeführt werden. Dieses wird bei der Selbstreinigung im Boden wie in fliessenden Gewïssern durch Mikroben bewirkt.

Es kann daher, wie der Erste von uns in seiner Schrift über „die Verunreinigung der Gewässer" ete. und in verschiedenen Abhandlungen des Oefteren hervorgehoben hat, nicht unter allen Umständen, wie die einseitigen Bakteriologen fordern, als einzige oder doch crste Aufgabe der Wasserreinigung angesehen werden, die Mikroben in denselben zu zerstören oder $\mathrm{zu}$ beseitigen; im Gegentheil wärde es in viclen Fällen von grösster Bedeutung sein, wenn die die organischen Stoffe zersetzenden Baktcricn reingezüchtet werden könnten, um womöglich dureh Zusatz von solchen Reinkulturen die Zersetzung der organischen Stoffe zu beschleunigen. Auch auf die Bedeutung der Sauerstoffzuführung, sei es durch I üftung oder Filtration der gefaulten Wässer, behufs Mineralisirung (Oxydation) der Fiulnissstoffe labe ich wiederholt hingewiesen.

Die neucrdings von W. J. Dibdin, J. Chemiker des Grafschaftstaths von London, und vom Kulturtechniker W. Sch weder in Gross-Lichtcrfelde bei Berlin vorgeschlagenen Verfahren gehen von diesen an sich ganz richtigen Gesichts-

1) Centralbl. der 13:tururwatumgr 1897,, . 453.

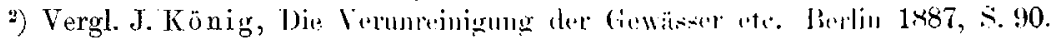


punkten aus und fragt sich nur weiter, ob die Verfahren schon diesem Zweck entsprechen oder unter welchen Verhältnissen sie anwendbar und empfehlenswerth sind.

Bis jetzt sind die anf vorstehendem Grundsatz beruhenden Verfahren unseres Wissens in Exeter und Sutton in England nach Dibdin, sowie in Gr. Lichterfelde bei Berlin nach Schweder versuchsweise eingerichtet, und haben wir die Wirkung derselben durch Untersuchung der ungereinigten und in verschiedenen Stadien gereinigten Abwässer festzustellen gesucht. Die Wasserproben wurden uns in zuvorkommendster Weise von den ersten beiden Reinigungsanlagen durch Herrn Ingenieur H. Alfred Röchling in Leicester, von der letzteren Reinigungsanlage durch Herrn Kulturtechniker W. Schweder übermittelt. Da alle 3 Reinigungsanlagen im Princip zwar gleich, aber in der Ausführung etwas verschieden sind, so mögen sie hier alle 3 kurz beschrieben werden.

a) Die Reinigungsanlage in Exeter. Dieselbe hat nach einer von Ingenieur Röehling angefertigten und uns überlassenen Skizze (Fig. 13) folgende Anordnung:

Die Spüljauche der Stadt, angeblich vorher präparirt ${ }^{1}$ ), tritt bei $a_{1}$ und $a_{2}$ (Sandfänge) in das Iuftdicht bedeckte sog. septische Becken A (Verflüssigungsbecken), in welchem sie bei einer Durchflussgeschwindigkeit von ungefähr $0,22 \mathrm{~m}$ pro Sekunde durchschnittlich 21 Stunden verbleibt, um dann in den mit einem Lüfter versehenen Vertheilungsbrunnen $B$ übergeführt zu werden. Von hier aus gelangt das Beckenabwasser in eines der Filter $F_{1}$ bis $F_{5}$, deren Betrieb intermittirend ist, $d$. h. nach Füllung bleibt das Filter eine Zeit lang voll stehen, wird dann schnell entleert und hat nach Entleerung eine volle Ruhepause.

Jedes der 5 Filter hat eine Oberfläche von 67 qm und eine Tiefe von $1,524 \mathrm{~m}$. Die Filtermasse besteht bei 4 Filtern vorwiegend aus zerkleinerter Schlacke von Kesselfeuerungen; deren Stücke einen Durchmesser von 3,2 bis $12,7 \mathrm{~mm}$ haben. Nur in einem Filter werden Koksstücke von gleichem Durchmesser wie die Schlackenstücke benutzt; doch hält Koks die Feuchtigkeit mehr zurück und wird in Folge dessen langsamer durchlüftet als Schlacke, weshalb er sich für die Filtration von Jauche nicht so gut eignet als letztere.

Die Wasserkapazität der Filter betrug anfänglich $60 \%$, ging aber wahrscheinlich in Folge Verschmutzung der Filter bald auf $38 \%$ herunter. Da die Zuflussmenge des städtischen Abwassers während 24 Stunden erheblich schwankt, so ist auch die Durchflussgeschwindigkeit durch die Verflüssigungskammer wie die Filtrationsgeschwindigkeit eine ungleichmässige - bei Tage ungefähr doppelt so gross als bei Nacht -, was ohne Zweifel nicht günstig ist.

Nach den in England über dieses Verfahren ausgeführten Untersuchungen wird der zur Oxydation der organischen Stoffe erforderliche Verbrauch an Kaliumpermanganat durch die Fäulniss, Lüftung und Filtration um ungefähr $80 \%$, das AlbuminoidAmmoniak um $60 \%$ in dem Filtrat vermindert. Nach Dibdin's Beobachtungen wird die Menge Ammoniak im Verflüssigungsbecken A vermindert, nach anderen dagegen vermehrt. Die Ablagerung von Schlick in demselben soll nur eine äusserst geringe

1) Wie und ob mit Chemikalien, wird geheim gehalten. 
scin; Röchling glaubt die Menge der als Schlick im Becken A niedergeschlagenen Schwebestoffe auf $20 \%$ veranschlagen zu können (vergl. weiter unten).

In dem Verflüssigungs- oder wohl richtiger Fïulnissbecken A sind ohne Zweifel vorwiegend anaërobe Bakterien thätig. Dieses ist, wie Röchling bcrichtet, daraus zu schliessen, dass sich in demselben brennbare Gase -- jedenfalls Sumpfgas - entwickeln, die sich in einem $\Lambda$ uer'schen Gasglühlichtbrenner anzünden liessen.
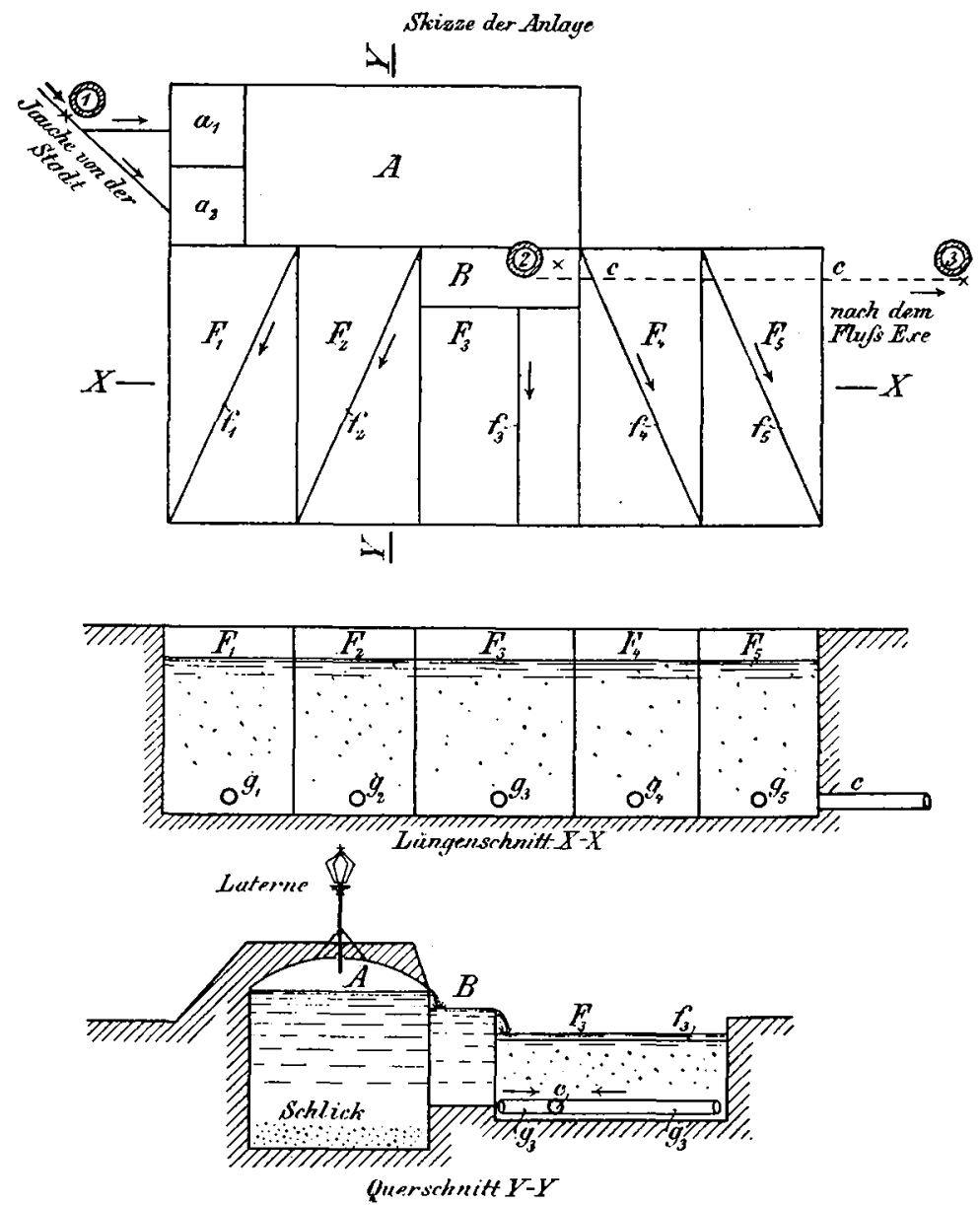

Fig. 13.

$\Lambda=$ Septic: T'ank, = (iailurungshecken, $=$ Verflüssigungshecken.

$a_{1}$ und $a_{2}=2$ kloine siandfïnge.

$B=$ Verthejlungslormmen mit Laifter.

"= Ianptontwilswerung der Filter nikch dem fluss Fxe.

$\mathrm{F}_{1}, \mathrm{~F}_{2}, \mathrm{~F}_{3}, \mathrm{~F}_{4}, \mathrm{~F}_{5}=$ Reinigumgshecken oder Filter.

$f_{1}, f_{2}, f_{3}, f_{1}, f_{5}=$ Vertheilungsrime der hehandelten Janche anf den Filtern.

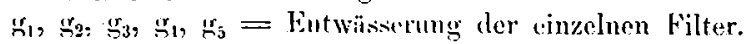

Das von den Filtern abfliessende gereinigte Wasser fliesst in Exeter nach dem Flusse Exe, worin es zur Zeit eines niedrigen Wasserstandes cine Verdünnung von 
1:40, also eine 40fache Verdünnung erfährt. An 21. Januar d. J., einem warmen Tage ohne Regenfall, hat Herr Röchling an den mit $x$ bexeichneten Stellen Proben entnommen und dazu bemerkt:

1. Probe: rohe Jauche in der Zeit voll $6 \mathrm{Uhr}$ morgens bis 2 l'hr nachmittags, jede halhe: Stunde entnommen; dieselbe war stark trübe und von geringem Jäulnissgeruch.

2. Probe: gefaulte Jauche heim Ausfiuss aus $\Lambda$ nach 24 stündigem Aufenthalt in Becken $\Lambda$; dieselbe wurde um 12 Uhr mittags auf einmal entnommen, weil durch den längeren Aufenthalt im Becken und durch die fortwährende Gasentrickelung eine innige Durchmischung angenommen werden konnte. Das Wasser war nur wenig getrübt und hatte, wie durchweg zu anderen Zeiten. einen starken Schwefelwasserstoff-Geruch.

3. Probe: Filtrat von den Filtern beim Ausfluss des Entwaisserungskanals $C$ in in die Exe in 4 Zeitabschnitten von 8 Uhr Vm. his 1,45, Jhr. Nm. Das Filtrat war zwar nicht getrübt, hatte aber zu Anfang der Entleerung eine stark gelbliche Farbe und einen schwach fäulnissartigen Geruch.

Die Prohen langten am 24. Januar, also 3 Tage nach der l'robenahme hier an und wurden sofort in üblicher Weise ${ }^{\text {) }}$ in Lntersuchung genommen.

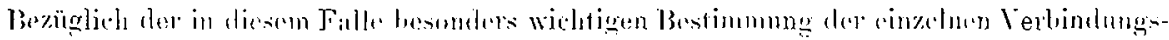

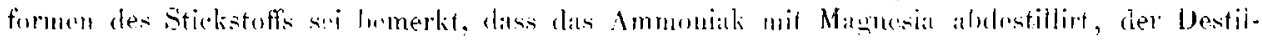

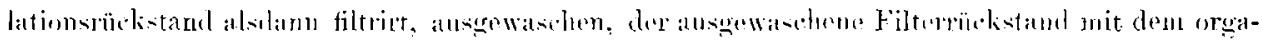

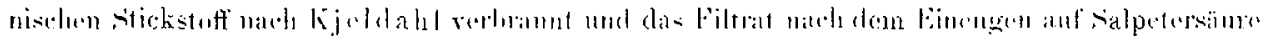

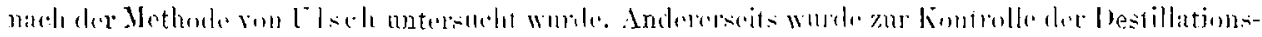

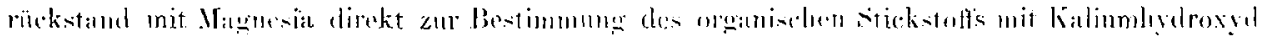

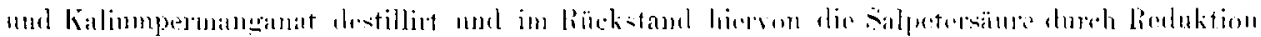

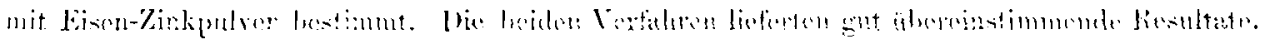

Die Untersuchung ergab für $1 \mathrm{I}$ :

\begin{tabular}{|c|c|c|c|c|c|c|c|c|c|c|c|c|c|}
\hline \multirow[b]{2}{*}{ Jauche: } & \multicolumn{2}{|c|}{ Schwebestoffe } & \multicolumn{2}{|c|}{ Gelöste Stoffe } & \multicolumn{2}{|c|}{$\begin{array}{c}\text { Zur Oxydation } \\
\text { erforderlicher } \\
\text { Sauerstoff }\end{array}$} & \multicolumn{3}{|c|}{ Stickstoff in Form von } & \multirow{2}{*}{$\begin{array}{c}\text { Schwe- } \\
\text { fel- } \\
\text { säure }\end{array}$} & \multirow[b]{2}{*}{$\begin{array}{l}\text { Phos- } \\
\text { phor- } \\
\text { säure } \\
\text { mg }\end{array}$} & \multirow[b]{2}{*}{ Chlor } & \multirow[b]{2}{*}{ Kal } \\
\hline & $\begin{array}{l}\text { orga- } \\
\text { nische } \\
\text { mg }\end{array}$ & $\begin{array}{l}\text { unorga- } \\
\text { nische } \\
\text { mg }\end{array}$ & $\begin{array}{c}\text { orga- } \\
\text { nische } \\
\text { (Glüh- } \\
\text { verlust) } \\
\text { mg }\end{array}$ & $\begin{array}{l}\text { unorga- } \\
\text { nisehe } \\
\text { (Gliub- } \\
\text { rüek- } \\
\text { stand) } \\
\text { mg }\end{array}$ & $\begin{array}{c}\text { in } \\
\text { saurer } \\
\text { Lö- } \\
\text { sung } \\
\text { mg }\end{array}$ & $\begin{array}{l}\text { in } \\
\text { alka- } \\
\text { lischer } \\
\text { Lö- } \\
\text { sung } \\
\text { mg }\end{array}$ & $\begin{array}{c}\text { organi- } \\
\text { sehen } \\
\text { Verbin- } \\
\text { dungen } \\
\mathrm{mg}\end{array}$ & $\begin{array}{c}\underbrace{\text { niak }}_{\text {niak }} \\
\mathrm{mg}\end{array}$ & $\begin{array}{c}\text { Sal- } \\
\text { peter- } \\
\text { săure } \\
\text { mg }\end{array}$ & & & & \\
\hline Rohe & 174,5 & 224,0 & 133,0 & 331,0 & 20,4 & 22,4 & 14,0 & 51,9 & 9,8 & 66,9 & 13,2 & 77,9 & 9 \\
\hline $\begin{array}{l}\text { 9. Gefaulte } \\
\text { 3. Golüftete }\end{array}$ & 53,5 & 67,5 & 124,0 & 290,0 & 17,0 & 19,6 & 3,9 & 32,9 & 14,0 & 58,0 & 7,7 & 81,4 & 105 \\
\hline 11. filtrirte & 61,0 & 61,0 & 201,0 & 299,0 & 8,4 & 16,4 & 3,9 & 9,3 & 17,1 & 63,1 & 5,1 & 77,9 & \\
\hline
\end{tabular}

In dem Filtrat No. 3 licss sich kolorimetrisch 1,0 $\mathrm{mg}$ salpetrige Säure nachweisen. In Cebrigen muss die Spüljauche als rerhältnissmässig stark verdünnt hezeichnet werden.

b) Reinigungsanlage in Sutton. Die Anordnung ist nach der von Ingenieur Röchling entworfenen Skizze (Fig. 14) folgende:

Der Reinigungsanlage in Sutton liegt derselbe Gedanke zu Grunde wie der in Exeter; sie unterscheidet sich wesentlich nur dadurch, dass das Fäulnissbecken B nicht wie in Exeter luftdicht verschlossen, sondern offen (unbedeckt) ist.

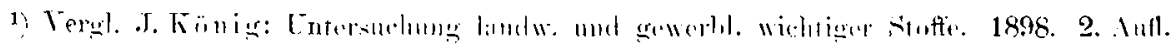
$\therefore 65111.1 \%$ 
Die rohe Janche geht erst durch Siebe, um grobfaserige Schwebestoffe zu entfernen, fliesst dann durch Kanal $A$ in das Becken B, worin sie 2 Stunden nach der Füllung verbleibt. Nach Verlauf dieser Zeit wird das Abwasser auf das Filter (oder Reinigungsbecken) $F$ durch Oeffnen des Schicbers $C$ abgelassen, worin sie ebenfals

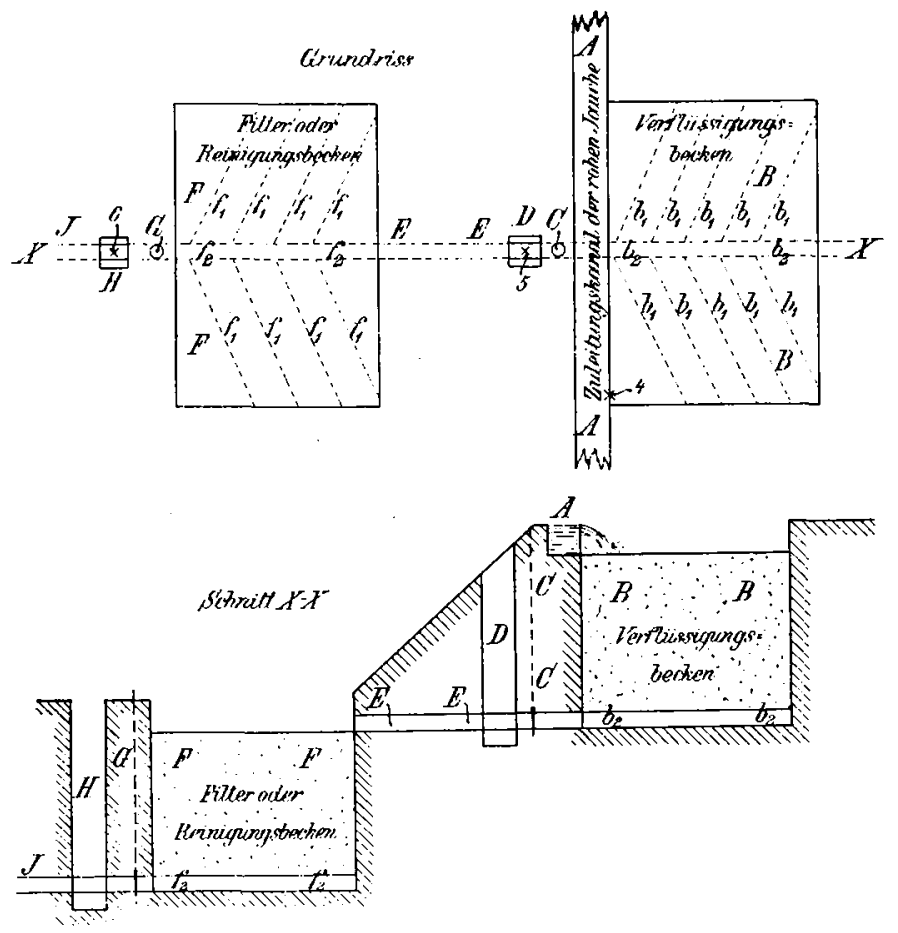

Fiị. 14.

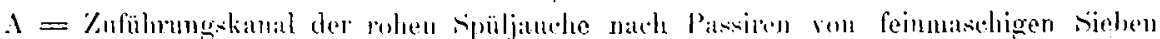

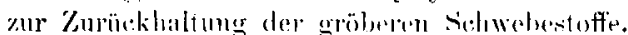

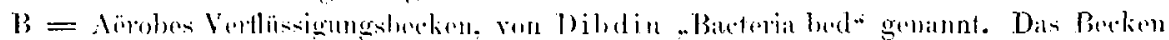
ist gan\% offict.

$l_{1}=$ die seitlichen lintwiksernngskanäle an Boden.

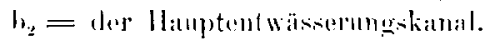

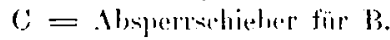

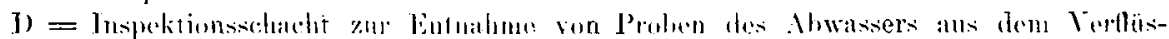
sigungshoecken li.

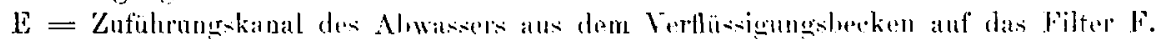

$r=$ Filter onder lininigungwhecken.

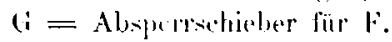

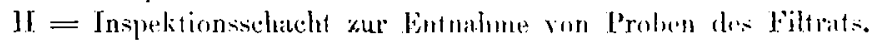

$J=$ Filtrataldasis anf"s Land oder direkt in den Flass.

$f_{1}=$ dis seitlichen Entwässerungrkintiile am Boden des Filters.

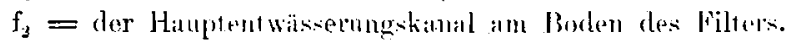

2 Stunden nach völliger Füllung verbleibt. Das Filtrat kann durch Schieber G entweder aufs Land oder in den Bach abgelassen werden. Nach dem Entleeren bleiben sowohl Becken B als Filter F leer stehen. Der Betrieb der Anlagen vertheilt sich daher wie folgt: 


\begin{tabular}{|c|c|c|c|c|}
\hline \multirow[b]{2}{*}{ Füllung eines Beckens } & \multicolumn{2}{|c|}{ Vurflïssignungsherken. } & \multicolumn{2}{|c|}{ lilter: } \\
\hline & & $45 \mathrm{Min}$. & & 90) Min. \\
\hline Vollstehen & $2 \mathrm{St}$. & - & $2 \mathrm{St}$ & - \\
\hline Entlecrung & $1 \mathrm{St}$. & $15 \mathrm{Min}$. & 1 St. & $15 \mathrm{Min}$. \\
\hline Leerstehen & $2 \mathrm{St}$. & & 2 St. & -- \\
\hline Zusammen & $6 \mathrm{St}$. & - & $5 \mathrm{St}$. & 35 Min. \\
\hline
\end{tabular}

Man ersieht hieraus, dass sowohl der Betrieb des Verflüssigungsbecken $\mathrm{B}$ wie der des Filters $\mathbf{F}$ intermittirend ist, und was die Gesammtfiltrationsgeschwindigkeit anbelangt, so dürfte dieselbe ungefähr $40 \mathrm{~mm}$ pro Stunde oder $1 \mathrm{cbm}$ pro qm in 24 Stunden betragen.

Da die Anlage nicht die ganze Spüljauche behandelt, sondern der Ueberschuss anderweitig gereinigt wird, so können die Verflüssigungs- und Reinigungsbecken am Tage wie in der Nacht gleichmässig arbeiten.

Die Konstruktion anlangend, so hat jedes Fäulniss- (Verflüssigungs-)Becken eine Oberfläche von $154 \mathrm{qm}$ und eine Tiefe von 1,066 m. Als Füllungsmasse werden gebrannte Thonstücke benutzt, deren Durchmesser grösser als $12,7 \mathrm{~mm}$ ist; die groben Stücke kommen auf den Boden, die kleineren nach oben; auf die Verhinderung der Staubbildung wird besondere Sorgfalt verwendet, weil der Staub die poröse Thonmasse verstopfen würde. Letztere soll $63 \%$ des Kubikinhaltes, die Jauche nur $37 \%$ desselben einnehmen.

Die Filter haben den gleichen Umfang wie die Fäulnissbecken, sind aber statt mit Thonstücken mit Koksstücken gefüllt. Die Entwässerung ist im Becken B wie in den Filtern $\mathrm{F}$ eine primitive, weil beim Oeffnen der Absperrschieber eine bedeutende Auslaugung der Filtermasse statthat.

Von dieser Anlage hat Herr Ingenieur Röchling am 22. Jan. 1898 an den mit $X$ bezeichneten Stellen ebenfalls Proben entnommen, die gleichzeitig mit denen von Ex eter bei uns eingingen. Leider sind diese Proben nicht während der ganzen Zeit des Aufenthaltes des Wassers in der Reinigungsanlage entnommen, sondern nur einmal, nämlich:

4. Probe: rohe Jauche, $12 \mathrm{Uhr}$ mittags ohne Einschluss vor Regenwasser, ziemlich frisch, ohne Fäulnissgeruch, aber stark trübe.

5. Probe: gefaulte Ja uche, Abwasser vom Fäulnissbecken B, mittags 11,45 Uhr, wolkig getrübt, gelblich gefärbt, von starkem Fäulnissgeruch (wie immer); die Jauche hatte ausnahmsweise nur 1 $1 \frac{2}{2}$ Stunde im Becken B gestanden.

6. Probe: Filtrat von den Filtern F, 12,15 Uhr mittags aus dem Inspektionsschacht $H$ entnommen, nur wenig getrübt, von blassgelblicher Farbe und ohne Geruch.

Die wie vorstehend ausgeführte Untersuchung ergab für 11 :

\begin{tabular}{|c|c|c|c|c|c|c|c|c|c|c|c|c|c|}
\hline \multirow[b]{2}{*}{ Jauche } & \multicolumn{2}{|c|}{ Schwebestoffe } & \multicolumn{2}{|c|}{ Gelöste Stoffe } & \multicolumn{2}{|c|}{$\begin{array}{c}\text { Zur Oxydation oxyer } \\
\text { erforderlicher } \\
\text { Sauerstoff }\end{array}$} & \multicolumn{3}{|c|}{$\begin{array}{c}\text { Stiekstoff in Form } \\
\text { von }\end{array}$} & \multirow{2}{*}{ 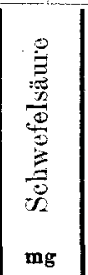 } & \multirow{2}{*}{ 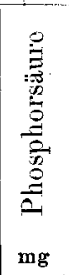 } & \multirow[b]{2}{*}{$\frac{5}{\partial}$} & \multirow[b]{2}{*}{ 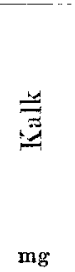 } \\
\hline & 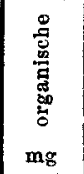 & 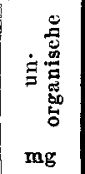 & 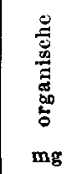 & 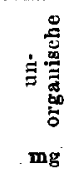 & 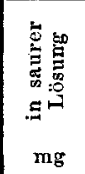 & 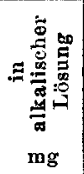 & 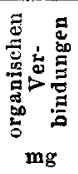 & 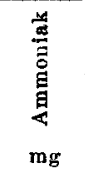 & 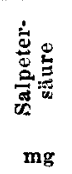 & & & & \\
\hline 4. Rohe & 336,0 & 201,0 & 234,0 & 579,0 & 40,0 & 44,8 & 9,3 & 100,1 & 11,4 & 100,8 & 25,6 & 70,8 & 225,0 \\
\hline $\begin{array}{l}\text { 5. Gefanlte } \\
\text { 6. Gelüftete }\end{array}$ & 57 &, 5 & 175,0 & 632,0 & 26,8 & 25,6 & 5,5 & $\mathbf{3 o ̄}, 0$ & 19,2 & 82,3 & 10,2 & 77,9 & 205,0 \\
\hline II. filtrirte & 13 & 3,5 & 224,0 & 624,0 & 11,2 & 14,6 & 3,9 & 4,7 & 23,8 & 125,5 & 4,5 & 74,3 & 220,0 \\
\hline
\end{tabular}


In dem Filtrat liessen sich kolorimetrisch nur Spuren, nämlich $0,2 \mathrm{mg}$ salpetrige Säure für 11 nachweisen.

Dieses Abwasser war somit viel stärker mit Jauchestoffen verunreinigt als das Abwasser von Exeter; wenngleich die 3 Proben keinem guten Durchschnitt entsprechen, so zeigen sie doch dieselben Beziehungen wie die Proben von Exeter.

c) Reinigungsanlage in Gross-Lichterfelde bei Berlin. Die nach vorstehendem Grundsatz von Kulturtechniker W. Schweder (Schweder \& Co.) in Gross-Lichterfelde bei Berlin, dem früheren Mitarbeiter von Alex. Müller, angelegte Reinigungs-Vorrichtung ist viel vollkommener eingerichtet als die englischen Anlagen in Exeter und Sutton. Die Art der Einrichtung erhellt aus nachstehender Zeichnung (Fig. 15).

Das Wasser tritt aus dem Kanal bei a in den Raum I (vergl. Fig. 15), welcher in halber Höhe mit einer Mauer durchsetzt ist, vor welcher das unter Druck eintretende Wasser behufs gleichmässiger Durchmischung und besserer Sedimentirung hochgestaut wird, und die an ihrer Oberkante eine Reihe eingemauerter kleinkalibriger Drainrohre enthält, durch welche das Wasser in den Leerraum zwischen I und II übertritt. Die korrespondirende Mauer ist bis unter die Bedachung hochgeführt, enthält dagegen am Boden eine Anzahl Drainrohre eingemauert, wodurch die Jauche in der Richtung der Pfeile in den Raum II tritt und aus diesem das vom vorigen Tage aufgespeicherte Wasser von unten auf verdrängt.

Die Räume I und II sind bedacht, um Licht- und Luftzutritt abzuhalten, ausserdem mit starken Mauern und einem Erdwall umgeben, um sie vor grossem Temperaturwechsel zu schützen; ausserdem sind sie mit einer Decke von Torfmull auf einem engmaschigen, unter den Balken ausgespannten Drahtnetz abgedeckt, welcher nicht nur einen schlechten Wärmeleiter bildet, sondern auch desodorirend wirkt.

In Raum II findet eine lebhafte Fäulniss statt und werden die Schwebestoffe, die schon theilweise in Raum I zurückgehalten werden, theils niedergeschlagen, theils gelöst (verflüssigt). Um eine Fortführung der Schwebestoffe (sei es der niedergeschlagenen, sei es der schwimmenden) zu vermeiden, sind in der dem Eintritt gegenüberliegenden Seitenwand Knierohre angebracht, deren wagerechter Schenkel im Höhenniveau der Jauche liegt, während der senkrechte etwa $1 \mathrm{~m}$ tief in die Jauche eintaucht, sodass weder die obenaufschwimmenden noch die am Boden befindlichen Schwebestoffe beim Austritt des Wassers aus der Faulkammer mitfortgeführt werden können.

Die aus dem Raum II austretende Jauche hat eine stark faulige Beschaffenheit. Um die Oxydation in den Filtern zu fördern, wird sie in Raum III dem Einfluss frischer Luft ausgesetzt oder gelüftet. In demselben sind mehrere Lochbleche stockwerkartig über einander angebracht, in einem vertikalen Abstande von $0,30 \mathrm{~m}$ von einander und jedes $15 \mathrm{~cm}$ mit Kies beschüttet, sodass zwischen jedem Lochblech ein freier Luftraum von $0,15 \mathrm{~m}$ Höhe verbleibt. Diese Lufträume sind durch die in die Umfassungswand eingesetzten Drainrohre mit einem schornsteinähnlichen Ventilationsschacht verbunden, die aus dem Raum II abfliessende gefaulte Jauche durchrieselt in vielen dünnen Fäden regenförmig die einzelnen Luftkammern und wird in diesen einer lebhaften Lüftung ausgesetzt. Während in der Faulkammer II nach den bakteriologischen Untersuchungen vorwiegend nur anaërobe Bakterien thätig sind, stellen sich in Raum III nach Aufnahme von Luftsauerstoff aërobe Bakterien ein und findet hier auch schon eine schwache Nitrifikation statt. 


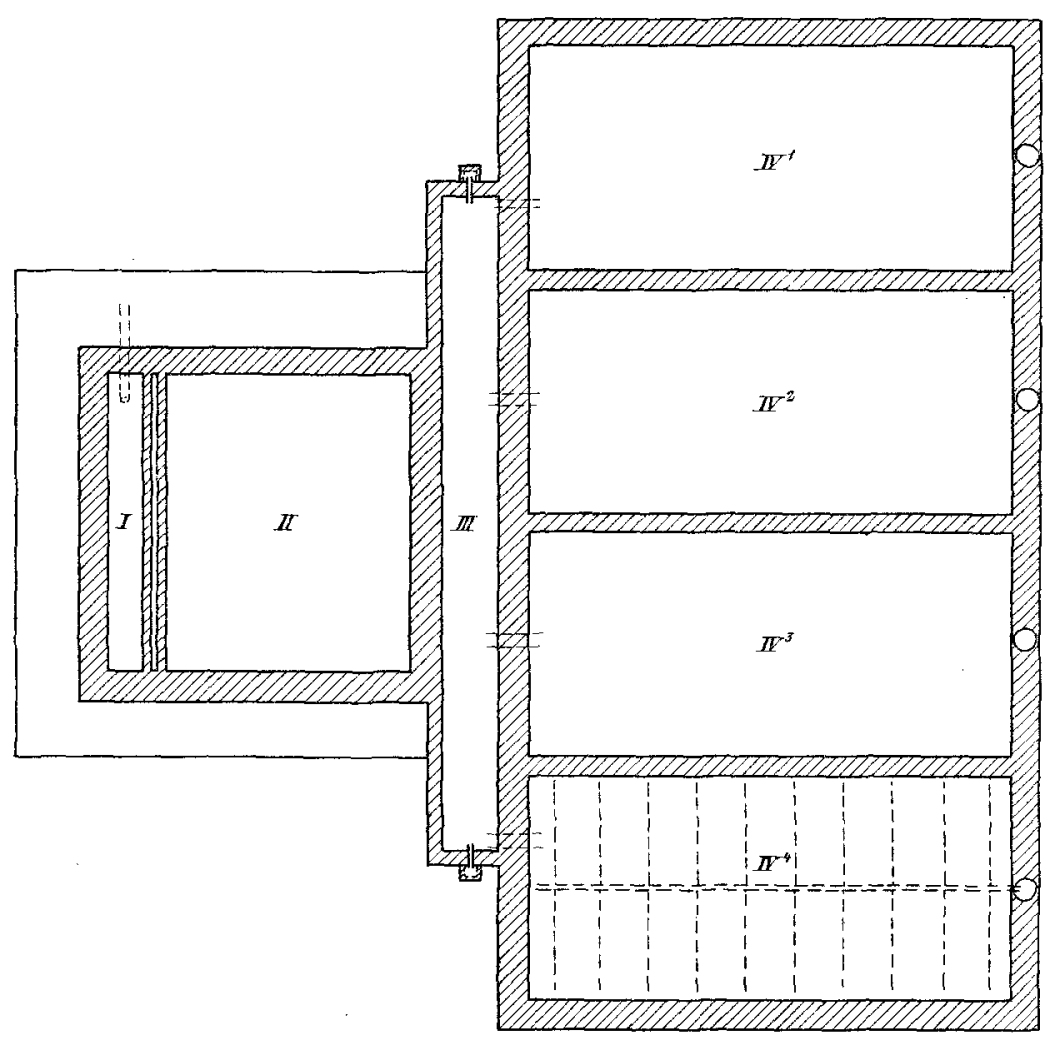

Grundrifs

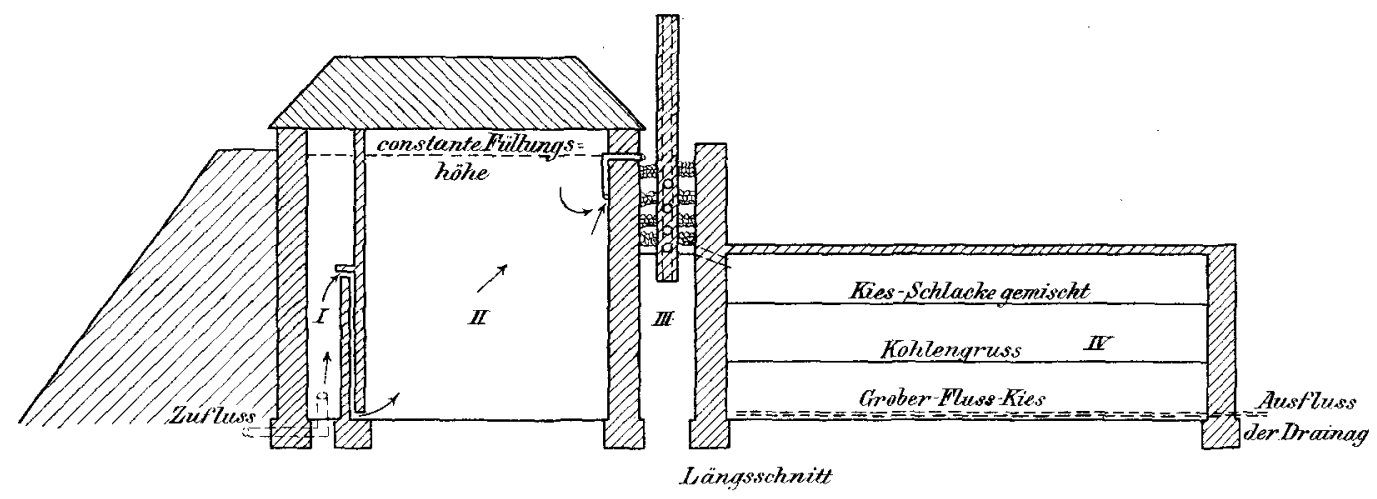

Fig. 15.

I and II Räume für Klärung und fanlige Gälırung.

III liam für Lüftung (Gradirwerk).

IV ${ }^{1--4}$ Oxydations - Filterraum.

IVt derselbe mit Ansicht der Untergrunddrainage. 
Die gelüftete Jauche sammelt sich unten in Raum III und kann zur weiteren Oxydation beliebig auf die einzelnen 4 Filter (Oxydationsraum IV) geleitet werden. Diese bestehen von unten nach oben schichtweise aus 0,30 m Kies, 0,75 m Koksgruss und 0,30 m Gemisch von feinem Kies und gekörnter Steinkohlenschlacke (vergl. Fig. 15). Die unterste Iage von Kies ist mit einer schwachen Beimengung von Muschelkalk vermengt.

Am Boden der Filter befindet sich eine einfache Ackerdrainage, deren Hauptsammelstränge (vergl. Fig. 15) durch ein Petersen'sches Ventil geschlossen werden können. Dic Füllung der Filter wird bei geschlossener Drainage vorgenommen und dauert bis zur vollen Sättigung 2 Stunden. Während der Füllung steigt die in den Filtern, besonders in dem porösen Kohlengruss eingeschlossene Luft durch die Jauche nach oben und erhöht die oxydirende Wirkung der Bakterien. Mit den Vollfüllen der Filter nach 2 Stundeu hat die Oxydation bezw. die Mikrohen-Thätigkeit ihren Höhepunkt erreicht; das Wasser wird dann nach Oeffnen des Petersen schen Ventils abgelassen und soll stets klar, geruchlos und frei von Schwefelwasserstoff sein.

Wenn das Filterabwasser auch als frei von Ammoniak bezeichnet wird, so trifft das für die von uns untersuchten Proben nicht zu (vergl. die nachstehenden Analysen).

Der Anmoniak- und organische Stickstoff wird aber grösstentheils in Salpetersäure und salpetrige Säure - das Verhältniss der letzteren ist schwankend - übergeführt. Seitdem die Filter (Oxydationsraum IV) ähnlich wie bei Mistbeeten mit Matten, welche die Abkühlung bei Frostwetter verhindern, die direkten Sonnenstrahlen abhalten, aber die Luftcirkulation nicht beeinträchtigen, bedeckt sind, soll die Nitratbildung das Uebergewicht haben.

Fine nerkbare Frwarmung findet im Faulraum II nicht statt, wohl aber in den Filtern, dem Oxydationsraum. Solbst bei einer Aussentemperatur von $-6^{\circ}$ hat hier die Wirmesteigerung in $4-5$ Stunden $2 \ldots 3^{\circ}$ betragen.

Das von den Filtern abfliessende Wasser enthält naturgemäss zahlreiche Balterien, vorwiegend nitrificirende neben anderen (u. A. Bacterium ('oli commune). Ein Versuch, diese durch Sandifltration zu entfernen, ist missgluickt, weil das Drainwasser keine Schwebestoffe enthält, um eine Schliekschicht auf den Filtern bilden zu können.

W. Schweder hat aber das Drainwasser mit Vortheil zur Berieselung einer Grastläche benutzt, ausserdem gefunden, dass es, in einen Feldtumpel geleitet, dem Gedeihen von Fischen durchaus nicht nachtheilig gewesen ist.

Auch W. Schweder hebt die geringe Schlammablagerung in dem Faulraum I und II hervor; seit Mai 1897 bis Mitte Januar 1898 haben ungeführ 20000 clom Jauche die Anlage durchlaufen, jedoch dürfte nach Schweder's Schätzung die Menge abgeschiedenen Schlammes höchstens 1 cbm betragen.

Auch der Oxydations-Filterraum zeigt, wie W. Schweder berichtet, keine wesentliche Veränderung.

An der Oberfläche ist sie zwar nacl jedesmaliger Benutzung bemerkbar, jedoch verschwindet dieselbe alsbald nach dem Abtrocknen der Oberfläche, was bei dom durchgeführten intermittirenden Betrieb täglich vorkommt.

Die Handhabung der 4 Filterräume ist nämlich folgende:

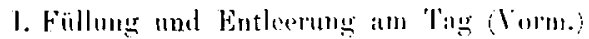

\begin{tabular}{|c|c|c|c|c|}
\hline \multicolumn{2}{|c|}{ Filterraum Fuüllung } & \multicolumn{2}{|c|}{$\begin{array}{l}\text { Verbleib im } \\
\text { Oxydations- } \\
\text { Eilterraum }\end{array}$} & \multirow{2}{*}{$\begin{array}{c}\text { Entleerung } \\
10-10 \% \text { Uthr }\end{array}$} \\
\hline$I_{1} \quad 6--8$ & Ehr & $8-10$ & Jhr & \\
\hline $8-10$ & - & $10-12$ & - & $12-12 \frac{1}{2}$ \\
\hline $\mathrm{IV}_{3} 10-12$ & - & $12-2$ & - & $2-21$ \\
\hline$I V, 12--2$ & - & $2-4$ & - & $4-4^{1 / 2}$ \\
\hline
\end{tabular}

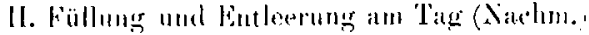

\begin{tabular}{|c|c|c|}
\hline Fullung & $\begin{array}{l}\text { Verbleib im } \\
\text { Oxydations- } \\
\text { Filterraum }\end{array}$ & Entleerung \\
\hline $2-4$ Jhr & $4-6$ L Lhr & $6-6^{:}:$UThe \\
\hline $4-6-$ & $6--8-$ & $8-8^{1} / 2=$ \\
\hline $6-8$ & $8-10$ & $10-10^{1 / 2}$ \\
\hline
\end{tabular}


Die 14 stïndige Betriebszeit in dem Oxydations-Filterraum ist mit Rücksicht darauf berechnet, dass in der Zeit von 8 Uhr abends bis 6 Uhr morgens nur wenig Spüljauche gefördert wird. Es hat daher jedes Filter zwischen der jedesmaligen Füllung am Tage $3 \frac{1}{2}$ Stunden und von der Zeit der Abendentleerung bis zur Füllung am nächsten Morgen 13., Stunden, also im Ganzen 17 Stunden Ruhe, die sich für die Erneuerung und Füllung mit Luft als ausreichend erwiesen hat.

Der Faulraum II kann nach den angegebenen Maassen rund $82 \mathrm{cbm}$ fassen, während täglich 100-120 cbm gereinigt werden, so dass die rohe Jauche unter Hinzurechnung des Vorraumes I ungefähr 24 Stunden aufgespeichert werden kann, ehe sie, durch nachtretende Jauche verdrängt, wieder austritt. Jeder Oxydations-Filterraum fasst $15-20 \mathrm{cbm}$, so dass durch diesen bei 7 maliger Füllung $105-140 \mathrm{cbm}$ gefaulte Jauche fliessen können. Im Ganzen hat der Raum $128 \mathrm{qm}$ Fläche, so dass bei $140 \mathrm{cbm}$ Jauche auf $0,9 \mathrm{qm} 1 \mathrm{cbm}$ Jauche entfällt. Auffallender Weise läuft aus den Drains kaum die Hälfte der Flüssigkeit $a b$, als in den Oxydations-Filterraum eintritt. Wenngleich während des Aufenthaltes des Wassers in diesem Raum und besonders während der Ruhezeit der Filter eine erhebliche Wasserverdunstung statthat, so kann doch hieraus allein wohl schwerlich ein solcher Wasserverlust erklärt werden; ein Theil des Verlustes muss wohl auch durch Versickerung verursacht sein.

Die Kosten anlangend, so berechnet W. Schweder dieselben für eine Anlage zur Reinigung von 120-160 cbm (Tagesproduktion von etwa 2000 Personen) auf $12000 \mathrm{M}$. und für die jährliche Unterhaltung, die keine besonderen Betriebskosten, sondern nur zeitweise einen Arbeitsmann zum Oeffnen und Schliessen der Drainventile erfordert, auf 40-50 Pfg. für den Kopf der Bevölkerung.

Herr Kulturtechniker Schweder hatte die Freundlichkeit, am 18. und 19. Januar d. J. Proben Wasser aus seiner Reinigungsanlage in den verschiedenen Stadien der Reinigung für uns zu entnehmen und zwar:

1. Probe: rohe Jauche aus Berlin bei 12 stündigem Zulauf jede halbe Stunde 24 mal entnommen und Proben gemischt.

2. Probe: Jauche aus dem Faulraum, bei 12 stündigem Zulauf jede $1 \frac{1 / 2}{2}$ Stunde 7 mal entnommen.

3. Probe: ans dem Lüftungsraum III, wie sie in den Oxydations-Filterraum IV übertritt, wie No. 2 entnommen.

4. Probe, Drainwasser, wie No. 2 entnommen.

Diese Proben wurden wie die vorstehenden untersucht und ergaben für 1 l die in der Tabelle S. 189 (oben) angegebenen Resultate.

Das filtrirte bezw. oxydirte (Drain-)Wasser vom 18. Januar enthielt nach der kolorimetrischen Methode 6,0 mg, das vom 19. Januar 7,5 mg salpetrige Säure in 11 . Hiernach ist die Bildung von Salpetersäure und salpetriger Säure in dem Filter-Oxydationsraum ersichtlich und bedeutend stärker als in den zu ungefähr gleicher Zeit entnommenen Proben der beiden Dibdin'schen Anlagen in England. Auch die Schwebestoffe sind bis auf unwägbare Mengen entfernt, während sich in den letzteren 2 Anlagen noch eine ganz geringe Menge Schwebestoffe nachweisen liessen. Der Verbrauch an Kaliumpermanganat zur Oxydation der in allen Fällen filtrirten Wässer ist auf rund $1 / 3$ vermindert, während die Schwefelsäure durch Oxydation von Schwefelverbindungen in England nur eine geringe, in Gross-Lichterfelde eine erhebliche Zunahme erfahren hat. 


\begin{tabular}{|c|c|c|c|c|c|c|c|c|c|c|c|c|c|}
\hline \multirow[b]{2}{*}{ Jauche: } & \multicolumn{2}{|c|}{ Schwebestoffe } & \multicolumn{2}{|c|}{ Gelöste Stoffe } & \multicolumn{2}{|c|}{$\begin{array}{c}\text { Zur Oxydation } \\
\text { erforderlicher } \\
\text { Sauterstoff }\end{array}$} & \multicolumn{3}{|c|}{$\begin{array}{c}\text { Stickstoff } \\
\text { in Form von }\end{array}$} & \multirow{2}{*}{$\begin{array}{c}\text { Schwe- } \\
\text { fel- } \\
\text { säure }\end{array}$} & \multirow{2}{*}{$\begin{array}{l}\text { Phos } \\
\text { phor- } \\
\text { säure }\end{array}$} & \multirow[b]{2}{*}{ Chlor } & \multirow[b]{2}{*}{ Kalk } \\
\hline & $\begin{array}{l}\text { orga- } \\
\text { nische } \\
\text { mg }\end{array}$ & $\begin{array}{c}\text { unorga- } \\
\text { nische } \\
\text { mg }\end{array}$ & $\begin{array}{c}\text { orga. } \\
\text { nische } \\
\text { (Gliuh- } \\
\text { verlust) } \\
\text { mg }\end{array}$ & $\begin{array}{l}\text { nnorga- } \\
\text { nische } \\
\text { (Glüh- } \\
\text { rïck- } \\
\text { stand) } \\
\text { mg }\end{array}$ & \begin{tabular}{|c|} 
in \\
saurer \\
Lös \\
$\mathrm{mg}$
\end{tabular} & $\begin{array}{c}\text { in } \\
\text { alka- } \\
\text { lischer } \\
\text { ung } \\
\text { mg }\end{array}$ & $\begin{array}{l}\text { orga- } \\
\text { nischen } \\
\text { Verbin- } \\
\text { dungen } \\
\text { mg }\end{array}$ & $\begin{array}{c}\text { Am. } \\
\text { moniak } \\
\text { mg }\end{array}$ & $\begin{array}{c}\text { Sal- } \\
\text { peter- } \\
\text { säure } \\
m g\end{array}$ & & & & \\
\hline
\end{tabular}

Proben rom 18. Januar 1898.

1. Rohe $\ldots \mid$\begin{tabular}{|l|l|l|l|l|l|l|l|l|l|l|l|l|l|l|l|l|l|}
1129,0 & 281,0 & 759,0 & 861,0 & 30,8 & 36,0 & 29,6 & 76,2 & 7,8 & 26,8 & 32,0 & 240,0 & 240,0
\end{tabular}

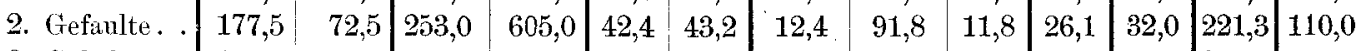

\begin{tabular}{l|l|l|l|l|l|l|l|l|l|l|l|l|l} 
3. Gelüftete . & 114,0 & 43,0 & 231,0 & 637,0 & 36,8 & 42,0 & 14,0 & 90,1 & 14,8 & 37,7 & 27,7 & 221,3 & 105,0 \\
4. Oxydirte u. & & & & & & & & & &
\end{tabular}

filtrirte . Spu \begin{tabular}{|l|l|l|l|l|}
\hline 325,0 & $\mathbf{1 0} 82,5$ & 9,6 & $\mathbf{1 5 , 6}$ & $\mathbf{7}, 0$
\end{tabular}

14,0

Proben rom 19. Januar 1898.

1. Rohe . . . $\mid$\begin{tabular}{l|l|l|l|l|l|l|l|l|l|l|l|l|}
1513,0 & 2562,0 & 272,0 & 653,0 & 38,4 & 40,0 & 28,8 & 79,2 & 10,1 & 41,2 & 44,4 & 221,3 & 225,0
\end{tabular}

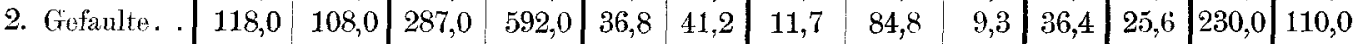

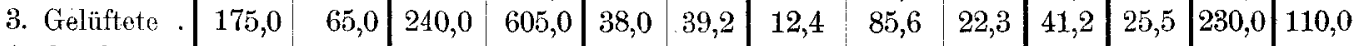

4. Oxydirte u

filtrirte

$$
\text { Spur }
$$

Auch bei diesen Versuchen nimmt wie oben bei den Polarite-Versuchen die Menge der gesammten gelösten Stoffe besonders an unorganischen Stoffen (Glührückstand) mehr oder weniger. zu, was darauf zurückgeführt werden muss, dass die durch die Oxydation bewirkte Bildung von Kohlensäure, Salpeter- und Schwefelsäure lösend auf mineralische Bestandtheile (kohlensauren Kalk) der Filtermasse wirken.

In der Fäulnisskammer hat in England eine erhebliche Abnahme, in GrossLichterfelde eine kleine Zunahme an Ammoniak stattgefunden. Letzteres ist das Wahrscheinlichere, weil die in Folge der verstärkten Fäulniss bewirkte Verflüssigung organischer Schwebestoffe eine Vermehrung des Ammoniaks bedingt, wenn nicht auch durch anaërobe Bakterien eine Entbindung von freiem Stickstoffgas statthaben soll. Nach den vorstehenden Analysen wurde gefunden:

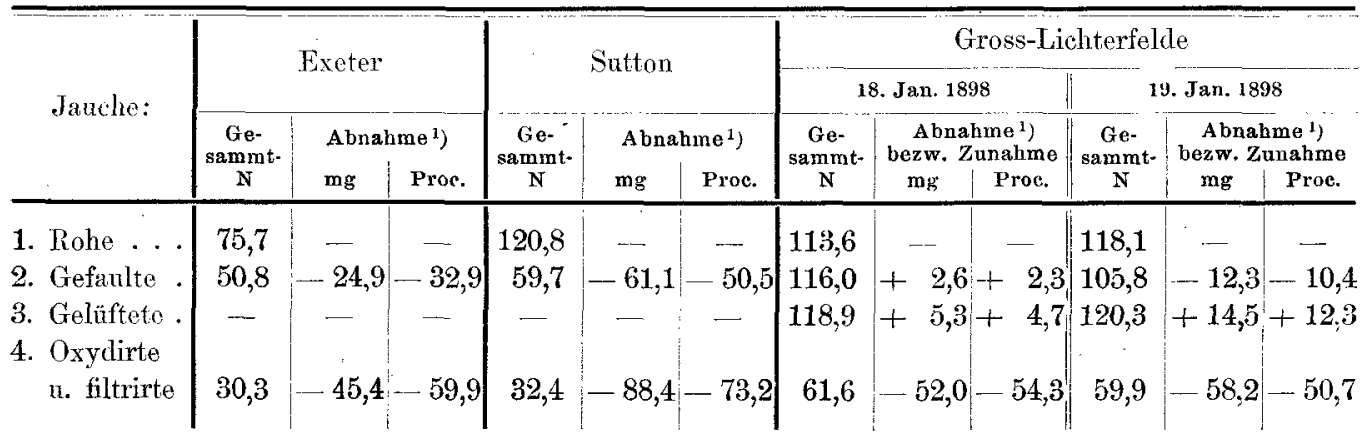

Ohne Zweifel beruht ein grosser Theil dieser Unterschiede im GesammtStickstoffgehalt darauf, dass die in den einzelnen Stadien der Reinigung ent-

1) Abnahme (-) bezw. Zumahne (+) gegen No. 1. 
nommenen Proben nicht vollständig der entnommenen Rohjauche entsprochen haben. Indess kann hieraus die grosse Differenz im Gehalt an Gesammt-Stickstoff zwischen der rohen und der von den Filtern abfliessenden gereinigten Jauche allein nicht erklärt werden, zumal der Gehalt an Chlor, als dem beständigsten Theil der Spüljauche, in den einzelnen Proben nur eine geringe Abweichung zeigt.

Es muss also auch aus diesen Versuchen geschlossen werden, dass bei der Oxydation (Nitrifikation) in den Filtern ein mehr oder weniger erheblicher Theil des gebundenen Stickstoffs in Form von freiem Stickstoff sich verflüchtigt.

Aus den bis jetzt gewonnenen Ergebnissen lassen sich folgende Schlussfolgerungen ableiten:

1. Das Ferrozone-Polarite-Verfahren bietet, was den 2. Theil des Verfahrens, die Filtration durch Polarite, anbelangt, keine Vorzüge vor anderen Filtrirmitteln; der gebundene Sauerstoff des Eisenoxyds hat keinen Einfluss auf die Grösse der Nitrifikation; in einem Filter aus Boden oder Koks ist dieselbe ebenso gross oder noch grösser.

2. Dem von Dibdin in London und Schweder in Gross-Lichterfelde eingeführten abwechselnden Fäulniss- und Oxydations-Verfahren liegt der schon mehrfach verfolgte ar sich richtige Gedanke zu Grunde, die organischen Stoffe, welche leicht in Fäulniss übergehen und sich weder durch chemische Fällungsmittel noch durch mechanische Hülfsmittel (Absetzenlassen oder Filtration) entfernen lassen, durch eine erhöhte Mikrobenthätigkeit zu zerstören und zu mineralisiren d. h. in Sauerstoffverbindungen überzuführen, in welcher Form sie nicht oder weniger schädlich für die Verunreinigung von Gewässern sind als im nicht oxydirten Zustande. Erreicht wird dieses einerseits durch Fäulniss-, andererseits durch nitrificirende Bakterien, also durch dieselben Lebewesen, welche auch bei der Selbstreinigung des Bodens und der Flüsse mitwirken.

3. Die erhöhte Fäulniss wird dadurch erzielt, dass das frische Abwasser in vor Luftzutritt geschützten Räumen zu einem Rest von bereits in starker Fäulniss begriffenen Jauche tritt und hier bis 24 Stunden verbleibt. Die eintretende starke Fäulniss hat zunächst eine vermehrte Ammoniak-Bildung aus organischen Stickstoff-Verbindungen zur Folge; wenn die gefaulte Jauche trotzdem eine geringere Ammoniakmenge zeigen sollte, wie Dibdin behauptet, so ist das, weil eine wesentliche Verdunstung von Ammoniak als solchem nicht angenommen werden kann, nur möglich, wenn bei der Ammoniakbildung durch anaërobe Bakterien auch freies Stickstoffgas entsteht. Ob letztere solche Wirkung überhaupt äussern, bleibt noch zu erforschen, wie ebenso, ob ein offener Fäulnissbehälter wie der in Sutton in derselben Weise wirkt, wie die geschlossenen Behälter.

4. Bei dem mehrstündigen Aufenthalt der Jauche in der Fäulnisskammer senken sich die specifisch schwereren organischen und unorganischen Schwebestoffe mehr oder weniger zu Boden; die organischen Stoffe unterliegen durch die fortgesetzte Fäulniss in dem Bodenschlamm einer allmählichen Zersetzung und Verflüssigung bezw. Vergasung und ist hieraus erklärlich, dass sich auf 
diese Weise nur verhältnissmässig wenig Schlamm in dem Fäulnissbehälter ansammelt. Wenn aber behauptet wird, dass sich bei diesem Verfahren überhaupt kein Schlamm bildet, so ist das übertrieben und jedenfalls der Wirklichkeit nicht entsprechend. Denn dass auch die unorganischen Schwebestoffe der Jauche, die unter Umständen nicht gering sind, verschwinden sollten, ist nicht möglich; ein solches eisernes Verdauungsvermögen kann man den Bakterien nicht zutrauen. Auch manche harten und grobfaserigen organischen Schwebestoffe der Jauche dürften kaum in kurzer Zeit zersetzt werden können. Wenigstens ergab eine aus dem Färulnissraum in Exeter von Herrn Ingenieur Röchling eingesandte Schlammprobe eine nicht unerhebliche Menge noch vorhandener stickstoffhaltiger Stoffe, nämlich in der Trockensubstanz:

\begin{tabular}{|c|c|c|c|c|c|c|c|}
\hline $\begin{array}{l}\text { Organische } \\
\text { Stoffe }\end{array}$ & $\begin{array}{c}\text { mit } \\
\text { Stickstoff }\end{array}$ & $\begin{array}{l}\text { Mineral- } \\
\text { stoffe }\end{array}$ & Kollk & $\begin{array}{l}\text { Phosphor. } \\
\text { säure }\end{array}$ & $\begin{array}{l}\text { Schwefel- } \\
\text { säurce }\end{array}$ & Kalì & $\begin{array}{l}\text { Sand und } \\
\text { Thon }\end{array}$ \\
\hline $32,37 \%$ & $2,86 \%$ & $67,63 \%$ & $4,69 \%$ & $0,79 \%$ & $2,38 \%$ & $1,47 \%$ & $39,75 \%$ \\
\hline
\end{tabular}

5. In Folge der Lüftung einerseits im Lüftungsraum, andererseits im Oxydations-Filterraum findet eine starke Oxydation der organischen Stoffe und Nitrifikation des Ammoniaks unter Entbindung von freiem Stickstoff statt; die Nitrifikation dürfte aber wohl in den seltensten Fällen eine vollständige sein. Das Vorhandensein von salpetriger Säure deutet an, dass die Lüftung bezw. Sauerstoff-Zuführung keine genügende war und wenn dann noch gleichzeitig Ammoniak und organische Stoffe in deutlicher, wenn auch in wesentlich verminderter Menge vorhanden sind, so kann bei trägem Lauf des Drainwassers in dicker Schicht immerhin wieder von Neuem Fäulniss eintreten, zumal dasselbe nach anderweitigen Untersuchungen eine grosse Anzahl von Bakterien enthält. Diese brauchen nicht pathogener Art zu sein, um noch schädlich wirken zu können; es ist. vielmehr anzunehmen, dass bei dem starken Ueberwiegen der Fäulniss- und nitrificirenden Bakterien die pathogenen. Keime verdrängt oder abgetödtet bezw, unschädlich gemacht werden, aber ein an Bakterien irgend welcher Art reiches Wasser mit genügend organischen Stoffen neigt an sich leicht zur Fäulniss.

Der Umstand, dass das Drainwasser in offenen Glasschalen nach mehrtägigem Stehen keine Fäulniss, überhaupt keine sichtbare Veränderung zeigt, ist noch kein Beweis gegen diese Annahme. Denn auch das stärkste Schmutzwasser unterliegt in offenen flachen Schalen mehr oder weniger schnell einem Selbstreinigungs- (Klärungs-) Vorgang, indem sich der faulige Geruch alsbald verliert, während es bei Einleitung in Flüsse mit geringer Stromgeschwindigkeit und mangelhaftem Luftzutritt alsbald Fäulniss hervorruft. Diese Art Prüfung, die vielfach von den Verwaltungsbehörden vorgeschrieben und geübt wird, hat schon häufig zu irrigen Schlussfolgerungen Veranlassung gegeben.

6. Die Frage, ob dieses Reinigungsverfahren genügen wird, um eine Verunreinigung von fliessenden Gewässern in nachtheiliger Weise zu verhindern, hängt ganz von den örtlichen Verhältnissen, von der Menge und Stromgeschwindigkeit des Bach- oder Flusswassers ab. Ohne Zweifel braucht bei richtiger 
Handhabung des Reinigungsverfahrens das Drainwasser, um völlig unschädlich zu werden, durch ein Bach- oder Flusswasser nicht so stark verdünnt zu werden, als bei Einlass der rohen oder nur geklärten Jauche. Aber eine genügende Verdünnung von mindestens etwa $1: 10$ bis 15 bei mittelguter Stromgeschwindigkeit wird auch hier gefordert werden müssen, vorausgesetzt, dass die Thätigkeit der Fäulniss- wie nitrificirenden Bakterien wie der Gr. Lichterfelder Anlage anhält und nicht mit der Zeit oder zu gewissen Zeiten erlahmt.

7. Bis jetzt sind beide Verfahren nur für verhältnissmässig geringe Mengen Schmutzwässer eingeführt und hierfür scheinen sie, wenn von einer wirthschaftlichen Ausnutzung derselben abgesehen wird, am Platze zu sein. Auch können sie vielleicht dort gute Dienste leisten, wo eine Bodenberieselung nothwendig, aber kein geeigneter, d. h. stark luftiger, gut filtrirender Boden vorhanden ist; hier kann die Voroxydation die reinigende Wirkung des Bodens wesentlich unterstützen und sind alsdann geringere Bodenflächen für die Berieselung nothwendig.

8. Bei grossen Mengen Abwasser dagegen (von 30-50000 Einwohnern und mehr) erscheint die Anlage zu theuer und der Erfolg zu zweifelhaft. Nach W. Schweder kostet die Anlage für den Kopf der Bevölkerung 6 M. und in der jährlichen Unterhaltung 40-50 Pf.; dazu kommen aber noch die Kosten für eine Rieselfläche; denn ohne eine Nachrieselung mit dem Drainwasser dürfte es bei dem Verfahren, wenn es sich um die Abgänge von 30-50.000 Einwohnern und mehr handelt und wenn nicht äusserst günstige Flussverhältnisse für Aufnahme des gereinigten Abwassers vorhanden sind, auf die Dauer entschieden nicht abgehen, wenn auch die Rieselfläche mit Voroxydation wesentlich kleiner und billiger ausgeführt werden kann als ohne Voroxydation. Schweder selbst hält für seine kleine Versuchsanlage in Gross-Lichterfelde eine Nachrieselung mit dem Drainwasser für wünschenswerth, wenn auch nicht für nothwendig. Hat man aber einen geeigneten Boden in genügender Ausdehnung für Berieselung zur Verfügung, dann kann man die Oxydation der organisehen Stoffe auch der reinigenden Kraft des natürlichen Bodens überlassen und die Vorreinigung als zu theuer und wirthschaftlich unrichtig ganz umgehen. Denn die reinigende Wirkung ist dieselbe, ob man die Jauche durch die vorstehenden Kies-KoksFilter oxydirt und filtrirt oder durch den Boden. Auf 100 Kopf der Bevölkerung soll, wenn die Reinigung der Spüljauche durch Berieselung eine erfolgreiche sein muss, mindestens 1 ha Rieselfeld entfallen; für 100 Einwohner müssen, wenn deren Abgänge in vorstehender künstlicher Filtrationsanlage gereinigt werden sollen, $600 \mathrm{M}$. Anlagekosten aufgewendet werden, die nichts aufbringen, sondern sich nur abnutzen. Wendet man dieses Geld aber zum Ankauf von Boden an, so verzinst es sich wenigstens zum Theil und bei richtiger Ausführung der Rieselung sogar ganz. Dazu kommt aber noch der grosse wirthschaftliche Verlust an Stickstoff und sonstigen Pflanzennährstoffen bei der Oxydation und Filtration durch Kunstfilter allein. Solche Verluste sind auch bei der Bodenberieselung vorhanden, aber in viel geringerem Maasse, da die mine- 
ralisirten, d. h. oxydirten Bestandtheile der Jauche sofort v on den Nutzpflanzen aufgenommen und verwerthet werden.

Aus dem Grunde kann grundsätzlich kein Verfahren für Reinigung von Spüljauche dem durch Bodenberieselung Stand halten.

Um aber hierdurch den richtigen Erfolg zu erzielen, sind verschiedene Bedingungen zu erfüllen, nämlich:

a) Der für die Berieselung ausgesuchte Boden muss die geeignete Beschaffenheit haben. Lockerer, gut durchlässiger Sandboden ist am geeignetsten; wo die Durchlässigkeit zu wünschen übrig lässt, muss sie durch Drainage und künstliche mechanische Hülfsmittel unterstiutzt werden.

b) Es muss eine genügende Bodenfläche zur Verfügung stehen. Im Allgemeinen soll man dem Boden nicht mehr Spüljauche zuführen, als Stickstoff zur Deckung des höchsten Bedarfs einer landwirthschaftlichen Nutzpflanze darin vorhanden ist. Das sind im Mittel 100-120 kg Stickstoff und diese pflegen in 1000-1200 cbm Spüljauche, oder in den Abgiingen von 80-100 Personen vorhanden zu sein. Man soll daher die Rieselfläche so bemessen, dass auf böchstens 100 Kopf der Bevölkerung je 1 ha Riesclfirche entfïllt. Mleistens kommen aber auf 1 ha die Abgäuge von $200-400$ und mehr Personen und ist von selbst einleuchtend, dass der Ueberschuss an Stickstoff bezw. Stickstoffverbindungen, da sie auf dic Dauer nicht vom Boden festgehalten und gebunden werden, ins Drainwassel übergehen und entweder wieder das Grundwasser oder das Vorfluth-Flusswasser veruureinigen.

c) Die Rieselanlage muss einheitlich und so mit Gefälle angelegt werden, dass einerseits die Spüljauche jeder\%eit gleichmässig über die ganz' Fliache vertheilt, andererscits das Rieselwasser wiederholt wieder benutzt worden kann. Im Winter und in Regenzeiten ist ein Ueberschuss, in Sommer dagegen, bezw. in der für die Pffanzen wasserbedürftigsten Zeit, ein Mangel an Spüljauche vorhanden. Man muss es daher in der liand haben, dic Spüljauche bei vorlıandenen grossen Mengen gleichmïssig über die ganze Rieselflïche ausbreiten, dagegen in der regenarmen, wärmeren Jahreszeit das Drainwasser von höher gelegenen Flächon auf niedriger gelegenen behufs Anfeuchtung wieder verwenden zu könnell.

d) Hei dem Leberschuss an Stickstoft in der Spüljauche muss noch gloichzeitig mit Phosphorsäure (in Form von Thomasphosphatmehl) und Kali (in Form von Kainit) beigedüngt wrorden. Das Verhältniss dieser 3 wichtigsten Pflanzennährstoffe beträgt naimlich im Durchschnitt:

\begin{tabular}{|c|c|c|c|}
\hline & Stinkitoff & Phosphorsitules & killi \\
\hline In den Pflanzenernten & 100 & 44 & 151 \\
\hline In der Spüljanche & 100 & 19 & 32. \\
\hline
\end{tabular}

Führen wir daher der Rieselfläche so viel Spüljauche zu, dass dadurch nur der Bedarf an Stickstoff gedeckt wird, so erhalten dic Nutzpfanzen nicht 
genügend Phosphorsäure und Kali; leitet man aber so viel Spüljauche zu, dass der Bedarf an letzteren beiden Nährstoffen gedeckt wird, so wird zu viel Stickstoff verabreicht, der alsdann nicht nur zum grössten Theil, sei es in Form von Ammoniak oder Salpetersäure, ins Drainwasser übergeht, sondern auch abnorm entwickelte Pflanzen erzeugt. Um daher einerseits eine völlige Ausnutzung und Reinigung der Spüljauche, andererseits normal entwickelte Nutzpflanzen zu erzielen, darf man einer Rieselfläche nur so viel Spüljauche zuführen, dass der darin enthaltene Stickstoff von den Nutzpflanzen aufgenommen und verarbeitet werden kann, und müssen gleichzeitig Phosphorsäure und Kali in Form künstlicher Dünger beigefügt werden.

Die mangelhaften Erfolge, welche man vielfach mit der Spüljauchenrieselung erzielt hat, haben vorwiegend darin ibren Grund, dass man diese und andere an sich ganz selbstverständliche Regeln nicht berücksichtigt.

Die vorstehenden Reinigungsversuche zeigen wiederum aufs Neue, wie wichtig die gute Durchlässigkeit der Filtermasse und die abwechselnde Lüftung derselben für die Reinigung der Spüljauche sind und diese beiden Punkte verdienen auch bei der Bodenberieselung volle Berücksichtigung. Auch entspringt aus den Versuchen der Nutzen, den eine vorherige Fäulniss oder gleichsam Aufsehliessung der Spüljauche hat. Schon jetzt pflegt man dieselbe behufs Niederschlagung der gröbsten Schwebestoffe und um die Filzbildung auf den Rieselfeldern zu vermeiden, vorher in Stauteichen abzuklären. Es bleibt nach den vorstehenden Versuchen zu erwägen, ob man diese Stauteiche nicht zweckmässig vergrössert und bedacht, um durch eine intensivere Fäulniss einerseits die Spüljauchenbestandtheile mehr aufzuschliessen, andererseits die Schwebestoffe noch mehr zu entfernen und so eine ausgiebigere Reinigung durch die Bodenfiltration zu erzielen.

Die Städte stellen sich naturgemäss auf den Standpunkt, ihren Unrath so einfach und billig wie möglich los zu werden. Alle künstlichen Mittel und Reinigungsverfahren, mit wie grosser Ueberzeugung sie auch anfänglich angepriesen worden sind, haben sich nicht bewährt und sind allmählich wieder eingeschlummert. Die Kosten standen und stehen allgemein nicht im Verhältniss zu den Erfolgen, weil es durchweg nur gelingt, die Schwebestoffe zu entfernen, nicht aber die ebenso schädlichen löslichen Stoffe. Das einzige Verfahren, welches bei richtiger Handhabung einen wirklich durchschlagenden und den relativ besten Erfolg auch für die gelösten fauligen oder fäulnissfähigen Stoffe hat und haben kann, ist - darüber sind wohl alle Sachverständigen jetzt einig - die Bodenberieselung, und diese kann einzig unter sonst geeigneten Verhältnissen einen wirthschaftlichen Nutzen gewähren, während alle künstlichen Reinigungsverfahren nur einen wirthschaftichen Verlust bedeuten.

Dort, wo durchaus kein geeigneter Boden in genügender Ausdehnung ohne Aufwand zu grosser Kosten zu beschaffen ist, wird man allerdings zu anderen Reinigungsverfahren seine Zuflucht nehmen und in erster Linie eine Trennung der Fäkalien von den sonstigen Schmutzwässern anstreben müssen. 
Erstere lassen sich auf pneumatischem Wege oder durch Abfuhr entfermen, und entweder durch direkte Verwendung zur Düngung oder durch Verarbeitung auf Poudrette unschädlich machen. Für die Reinigung der Spülwässer müssen dann andere Reinigungsverfahren angestrebt werden und dürfte hierfür das vorstehende Dibdin-Schweder'sche Verfahren in grecigneter Verbesserung nach meinen Erfahrungen die grösste Beachtung verdienen. In andcren Fällen, wo fliessende Gewässer mit genügender Stromgeschwindigkeit und hinreichender Wassermenge zur Verfügung stehen, wird man die Spülwässer auch direkt in dic (Hewässer ableiten und die Reinigung der selbstreinigenden Wirkung des fliessenden Wassers überlassen könneu. Jedenfalls giebt es keine allgemein gültige Regel für die Unschädlichmachung der städtischen Abfallstoffe; die Frage will örtlich geprüft und gelöst werden.

Leider aber wird die Frage der Reinigung städtischer Abwässer durch eine einseitige bakteriologische Auffassung vielfach verwirt und deren Lösung verzögert. Wenn beispielsweise von Baktcriologen verlangt wird, dass die gereinigten städtischen Abwässer nur 300 Keime von Mikrophytell baben sollen, so ist das vom wissenschaftlichen wie praktischen Standpunkt gleich unverständlich. Wir können aus der städtischen Spüljauche nach keinem Verfahren ein Trinkwasser machen, und daraus ein keimfreies Wasser herzustellen, ist ebenso unmöglich als unnöthig. Gewisse Bakterien und niedere Lebewesen sind geradezu die Bedingung für die Reinigung der Spüljauche und was die Gefahr der Ansteckung durch pathogene Bakterien anbelangt, dic ohne Zweifel fast $\mathrm{zu}$ jeder Zeit in derselben vorhanden sein werden, so haben die Erhebungen von W. Prausnitz, R. Pasquay, R. Virchow, Th. Weyl, A. Bernstein u. A. crgeben, dass dic beständig in den städtischen Kanälen wie auf Rieselfeldern beschäftigten Arbeiter weder zahlreicheren Allgemeinerkrankungen noch bäufigeren specifischen Infektionserkrankungen ausgesetzt sind als anderweitig beschäftigte Arbeiter. Man muss daher wohl annehmen und verschicdene Versuche hahen dieses erwiesen, dass die pathogenen Bakterien bei der Ljebcrmaclit der Fäulniss- und sonstiger nicht gesundheitsschädlicher Bakterien cinerseits im Kampf ums Dasein, andererseits beirn Ausbreiten in Flüssen oder auf Rieselfeldern unter dem Einfluss des Sonnenlichtes oder sonstiger selbstreinigender Ursachen alsbald zu Grunde gehen.

Wir sind schon seit Jahr und 'lag gewohnt, auf dem Gebict der Reinigung städtischer Abwässer, einer der brennendsten Tagesfrag‘ $n$, jeden Augenblick mit den verschicdenartigsten Vorschlägen unter den hochtönendsten Anpreisungen überrascht $\mathrm{zu}$ werden, die zwar sebr bald verstummen, aber immer in Folgc unsachlicher Beurtheilung zu nicht geringen unnützen Ausgaben Veranlassung geben. Die Stadtverwaltungen glauben aus Sparsamkeitsrücksichten gar zu gern, dass doch Einer mal den Stein der Weisen erfunden haben könne.

Wenn man bedenkt, dass wir die durch Sonnenwärme und Sonnenlicht bedingte Arbeit der pflanzlichen Lebewesen, welche zur vollen Unschädlichmachung der stiddtischen und ähnlichen Schmutzwässer mit organischen Abfall- 
stoffen nothwendig sind, durch künstliche Mittel wohl regeln und unterstützen, aber nie vollständig ersetzen können, dann wird man bei Beurtheilung solcher Vorschläge nicht fehlgehen. In minimis natura maxima. In der spaltenden und wieder aufbauenden Lebenstbätigkeit der niederen und höheren Pflanzen liegt eine grosse, unberechenbare Kraftquelle, welche uns die Natur umsonst bietet; sie $z u$ ersetzen, ist an sich nicht möglich, sie aber durch chemisch-physikalische Hülfsmittel aufzuwiegen, mit einem versehwenderischen Kraftaufwand verbunden.

\section{Referate.}

\section{Allgemeine analytische Methoden und Apparate.}

K. Wedenmeyer: Ein Wort zur Stickstoffbestimmung nach Kjeldahl-Gunning. - Chem. Ztg. 1898, 92, 21.

Der Vorschlag Gunnings, die Zerstörung der organischen Substanz nach Kjeldahl durch Zusatz von Kaliumsulfat $z u$ beschleunigen, ist vielfach geprüft und empfohlen, und seit 1893 auch vom Verband der landwirthschaftlichen Versuchsstationen Nord-Amerikas angenommen worden. Verf. hat nun abermals eine grosse Anzahl der an den landwirthschaftlichen Versuchsstationen am häufigsten zur Untersuchung gelangenden Stoffe, darunter recht schwierig aufzuschliessende, vergleichenden Bestimmungen unterzogen und kann dieses Verfahren, welches auf dem Zusatz von etwa 10-15 g Kaliumsulfat beruht, nur auf das Wärmste empfehlen, da damit eine ganz erhebliche Zeitersparniss erzielt wird. Bei den Analysen wurde nach den Vorschriften des Verbandes Deutscher Versuchsstationen die mit Phosphorsäure und Quecksilber versetzte Schwefelsäure benutzt und dieser auf $20 \mathrm{ccm}$ je $10 \mathrm{~g}$ Kaliumsulfat zugesetzt. Bei Substanzen, die stark schäumen, fügt man zweckmässig das Sulfat erst nach dem ersten Aufkochen zu. Ist die Flüssigkeit farblos geworden, so ist die Aufschliessung beendet und man kann, nachdem die Flamme entfernt ist, nach $10-15 \mathrm{Mi}$ nuten langem Stehen mit Wasser verdünnen und die Flüssigkeit in den Destillationskolben überführen, ohne dass Krystallausscheidung erfolgt. Zahlreiche Beleganalysen beweisen, dass bei gleichbleibender Genauigkeit (Differenzen meist nur Hundertstel Procente) durchschnittlich bei Anwendung von Kaliumsulfat eine halbe Stunde zur Zerstörung der organischen Substanz genügt, während ohne diesen Zusatz zwei Stunden und mehr erforderlich sind - und dass demzufolge eine Stickstoffbestimmung nach Kjeldahl-Gunning innerhalb einer Stunde vollständig beendet sein kann.

\section{J. Mayrhofer.}

A. Schneegans: Bestimmung des Oeles in Emulsionen. - Journ. d. Pharm. Elsass-Lothring. 1897, 321; Pharm. Ztg. 1898, 43, 15.

Die Bestimmung des Oelgehaltes von Emulsionen durch Ausschütteln mit Aether (mit oder ohne Säurezusatz) oder durch Extraktion des Trockenrückstandes giebt bekanntlich nur unsichere Resultate. Verf. empfiehlt daher ein Verfahren, welches, wenn auch etwas umständlich, doch sicher zum Ziel fübrt.

$300 \mathrm{Th}$. der Oelgummiemulsion werden mit je $50 \mathrm{Th}$. weissen Thones und groben Sandes eingedampft. Gegen Ende der Operation werden 50 Th. entwässertes Natriumsulfat zugesetzt und das Gemisch unter bisweiligem Rühren zur Trockne gebracht, 